Article

\title{
Anomaly Detection in a Logistic Operating System Using the Mahalanobis-Taguchi Method
}

\author{
Takumi Asakura ${ }^{1, *(D)}$, Wataru Yashima ${ }^{1}$, Kouki Suzuki ${ }^{2}$ and Makoto Shimotou ${ }^{2}$ \\ 1 Department of Mechanical Engineering, Faculty of Science and Technology, Tokyo University of Science, \\ Tokyo 278-8510, Japan; wataru.eagles@gmail.com \\ 2 TOYO KANETSU K.K., Tokyo 136-8666, Japan; suzuki052@toyokanetsu.co.jp (K.S.); \\ shimotou@toyokanetsu.co.jp (M.S.) \\ * Correspondence: t_asakura@rs.tus.ac.jp; Tel.: +81-4-7122-1413
}

Received: 14 May 2020; Accepted: 17 June 2020; Published: 25 June 2020

\begin{abstract}
Product delivery via logistic systems is becoming more efficient, rapidly and continuously bringing products to the customer. The continuous operation of logistic equipment, however, can lead to mechanical stoppages due to excessive use. To avoid system failures, fatigue in each part of the system should be monitored, enabling the accurate prediction of potential stoppages and thus promoting overall system efficiency. To date, various kinds of anomaly-detection methodologies have been proposed. Among them, the Mahalanobis-Taguchi method, which simply describes the extent of a failure using the Mahalanobis distance, has been utilized to detect changes in the mechanical condition of facilities. However, the technique has not yet been applied to anomaly detection in a logistic operating system. In this paper, anomaly detection using the Mahalanobis-Taguchi method targeting the operational characteristics of a large-scale vertical transfer system is proposed and the validity of the method is discussed. The calculation used to produce proper values of the Mahalanobis distance is first developed based on simple excitation using a shaker. Mahalanobis distances under conditions of continuous operation of the target vertical transfer system are then obtained; distances for the system in an artificially damaged condition are compared to values produced under normal conditions, and any significant increase is used as an indicator of a problem. The applicability of the approach to a case involving continuous long-term operation is discussed using a simulation in which the target vertical transfer system is in continuous operation over a two-year period.
\end{abstract}

Keywords: Mahalanobis-Taguchi method; Mahalanobis distance; logistic operating system; vibration acceleration

\section{Introduction}

Recent improvements in technologies related to IoT (Internet of Things) have made real-time monitoring of various types of structures possible. In the field of structural health monitoring, sensor-embedding and wireless-transfer technologies have been developed to monitor the structural integrity of buildings and civil engineering facilities. In the logistics industry, as product delivery has become more efficient and less costly, the working time required for inspection and repair necessitated by unexpected problems can be a major issue, especially for an automatic logistic system operating in a twenty-four-hour active environment. In a conventional management system involving mechanical devices, the majority of maintenance work is done by maintenance workers based on their experience and intuition related to anomaly detection, the timing of which is typically driven by a stoppage of the operation due to some mechanical problem that has already occurred. This can lead to non-negligible economic losses. Thus, it is important to detect a deterioration or change in the mechanical state of a logistic operating system early and accurately in order to predict the time of potential failure 
and to avoid stoppages of the system to the extent possible. To this end, various damage-detection algorithms [1] and their application to mechanical structures have been studied. A number of studies have been conducted on damage-detection methods for rotating equipment such as bearings [2] and gearboxes [3], the failure of which can cause an entire system to be shut down. When detecting damage of machinery, the use of physical data such as the mechanical ones including vibration and the sound, electrical, and thermal ones can be effective as a basic measure [4]. Using acoustic emissions [5] from damaged parts can also provide an essential clue in damage detection. In addition to such basic measures, a variety of methods for detecting damage features by using machine learning such as neural networks $[6,7]$ has been proposed in recent years. Zhang et al. validated the applicability of SIDL (shift-invariant dictionary learning), which is an extension of regular dictionary learning and makes each atom replicated and shifted at an arbitrary position within the signal [8]. Li et al. have suggested a useful method for nonnegative matrix factorization (NMF) to detect damage to a gearbox [9]. This investigation described a feature extraction and feature selection scheme for hybrid fault diagnosis of a gearbox based on $\mathrm{S}$ transform, nonnegative matrix factorization, and mutual information. Assaad et al. also proposed a method for detecting abrasion in gearboxes [10], in which a model-based technique for detecting wear in a multistage planetary gearbox used by lifting cranes was proposed, and established a vibration signal model which dealt with cyclostationary and autoregressive models. In each of these cases, estimating changes in mechanical conditions based on a large amount of accumulated physical data is necessary for effective anomaly detection. Agrawal et al. investigated previously proposed anomaly-detection methods based on data mining [11], grouping the techniques into three categories: classification, clustering, and a hybrid method combining the two. According to this scheme, classification corresponds to neural networks, support vector machines, or genetic algorithms used to classify an unknown condition as normal or anomalous by using a classifier learned with a large amount of physical data collected under normal and anomalous conditions. Presenting research examples of classification problems addressed by a machine-learning-based model, Iannace et al. have proposed a noise-prediction method using random forest regression [12] and a noise-detection method [13] for HVAC (Heating, Ventilation, and Air Conditioning) noises using recursive partitioning. However, such a method is difficult to apply in the absence of sufficient anomaly data or if various kinds of anomalous states can occur, since in both cases the opportunity for machine learning will be limited. On the other hand, clustering, which includes the K-nearest neighbor method, is based on the idea of classifying already known data into groups and of identifying any outliers beyond the range of the already categorized groups as anomalies. For example, the clack-detection methods based on clustering algorithms $[14,15]$ have been investigated.

While various methods have been proposed for outlier detection, using the Mahalanobis-Taguchi (MT) method [16,17], by which the operating state of mechanical equipment can be represented with a single scalar value, offers a powerful approach to anomaly detection. The MT method was originally developed in quality engineering and was applied in various engineering fields due to its versatility. In particular, although various studies have been conducted in the field of anomaly detection, Soylemezoglu et al. have produced a system using the MT method to detect bearing damage [18]. Shakya et al. have also proposed a method for monitoring the health of bearings online and for detecting their deterioration [19]. Balsamo et al. verified a method for detecting the state of damage to a structure using the Mahalanobis distance [20], targeting simple systems such as frame structures. Herein, first of all, the feature of the vibration caused in the mechanical system can be described by various kinds of feature values, e.g., the power spectral density obtained by the Wavelet analysis [21] and its statistical values such as the kurtosis and skewness [22]. However, if the changes of the mechanical condition are complicatedly reflected to the power spectral density of the vibration, it is quite difficult to detect the complex change of the frequency characteristics. In the MT method, the Mahalanobis distance, which describes how the anomaly vibration data differ from the normal one, can be calculated based on the multidimensional feature vectors related to each of the vibration data. Therefore, this method has an advantage to describe the multidimensional values such as the power 
spectral density with a single value. The multidimensional feature vector can be easily composed of the multiband power spectrum with optimal bandwidth, which can be calculated from the power spectral density obtained by Fourier or Wavelet transform. Herein, we can also reduce the degree of freedom of the feature value by exchanging the narrowband frequency characteristics into wideband ones such as the one-third octave band levels.

Although various anomaly-detection methods have been proposed as mentioned above, a few studies have applied them to logistic equipment such as horizontal belt conveyors. The on-line inspection method of conveyor belts by using machine vision has been investigated [23,24]. The longitudinal tear of the conveyor belt is investigated for detection by a multispectral visual method [25]. The remaining lifetimes of steel cord conveyor belts were observed by using magnetic sensors [26]. Although there are some research cases for these belt conveyors, almost all of them are detected by the sensors other than those by vibration. Furthermore, there are few cases related to the anomaly detection of cargo lifters that vertically convey the logistic products. In any case, the techniques for the anomaly detection of logistics systems have not been yet fully studied and have been still in development. One reason for the lack of research cases in this field is that the mechanical characteristics of these operating devices can be rather complicated; such equipment is often composed of a wide variety of machine elements. Notably, however, since the structure of logistic equipment can include relatively flexible structures such as thin beams or columns, its operating state may be sensitively reflected in the vibration characteristics of the structure. A detection method involving vibration would be difficult to apply using supervised machine learning because of the following reasons. Firstly, it is difficult to recover the labels for specific malfunctions. Secondly, the vibration characteristics change dynamically as a function of the operating situation. Thirdly, a variety of unknown anomaly states in the mechanical system have the possibility to happen, and it is difficult to cover all the possible cases in advance by the detection algorithm. Although it is difficult to collect labeled data for the supervised classification, it is possible to detect an acceleration in vibrations with a sensor and to use the measured acceleration directly; differences between the equipment's normal and anomaly states can be observed in its frequency characteristics as well as in the absolute value of the raw acceleration level.

As described, the accuracy of anomaly detection in a logistic operating system using the MT method can be substantially improved by considering not only the absolute vibration level that occurs during the operation of the equipment but also transitions in the frequency characteristics. Then, the MT method makes it possible to clearly and simply detect changes in state, as transitions in the vibration state can be expressed as a single scalar value. In this study, an anomaly-detection method using the MT approach and targeting the operational characteristics of a large-scale vertical transfer unit (hereinafter, a "lifter") is developed. Firstly, the applicability of the method for steady-state vibrations generated from a simple shaker was examined as a basic study. Then, after that, based on this finding, the properties of the lifter in normal and artificially damaged conditions were investigated through the vibration measurement results obtained by setting the vibration sensor on the surface of the lifter. From the calculation results of the Mahalanobis distances for fragment data acquired under three types of anomaly conditions, it was suggested that the operating state of the lifter can be estimated by using the differences of the Mahalanobis distances. Finally, the validity of the proposed method was discussed from the simulation results in which the lifter was continuously operated for a period of approximately two years and the mechanical condition of the lifter's guide wheel changed gradually from its normal state to the anomaly condition.

\section{Method}

To determine the state of vibration based on the MT method, the Mahalanobis distance (hereinafter, "MD") is calculated. The unit space generated from the measured data in the normal operational state is used to evaluate the MD measured in the anomaly state. The detailed scheme is described below. 


\subsection{Target Operation System of Anomaly Detection}

Figure 1 shows the components of the automatic storage system targeted for anomaly detection in this study. The general function of the system is to store products scheduled for shipping; storage is automatically managed in the rack section using the shuttle vehicles shown in the figure. To put the products into and to take them out of storage, a conveyor for horizontal movement and a lifter for vertical movement are used. In many cases, the racks and conveyors in the system can be replaced immediately if one or more of their parts fail. Repairing/replacing the lifter, however, is not so easy. The lifter is essential for vertically conveying products and for distributing them to the various storage layers. It is expected that it will deteriorate more quickly than the other system components because of following reasons: Firstly, the lifter operates continuously for $24 \mathrm{~h}$ every day. Secondly, it frequently repeats drastic acceleration/deceleration. Thirdly, loading and unloading products on the carriage of the lifter are continuously repeated. If a problem occurs in the lifter, because of following reasons, it can have a relatively large impact on the movement of products to the shelves in each storage layer. Firstly, because the mechanical elements in the lifter are closely related and joined with one another, it often takes time to replace them in the event of a failure. Secondly, the repair of the lifter requires much time compared to that of the horizontal belt conveyor because the lifter is located in quite a narrow space surrounded by tall partition walls and, therefore, it needs to be repaired by a few people. As a consequence, effective anomaly detection for this part of the system is particularly important; if potential failures in the lifter can be predicted in advance, it is possible to minimize the economic loss due to equipment stoppage. In this study, we developed a prediction technique that can detect lifter failure in advance. As shown in Figure 1, as the lifter moves up and down, the carriage on which the product is placed moves up and down with it; in the operation of the lifter, vibration propagates to the entire body, including the steel columns supporting the lifter and other individual parts. With the proposed method, the transmitted vibration is detected by an accelerometer placed at optimal points on the body and changes in the vibration characteristics are effectively captured. Details of the method are described in the next section.

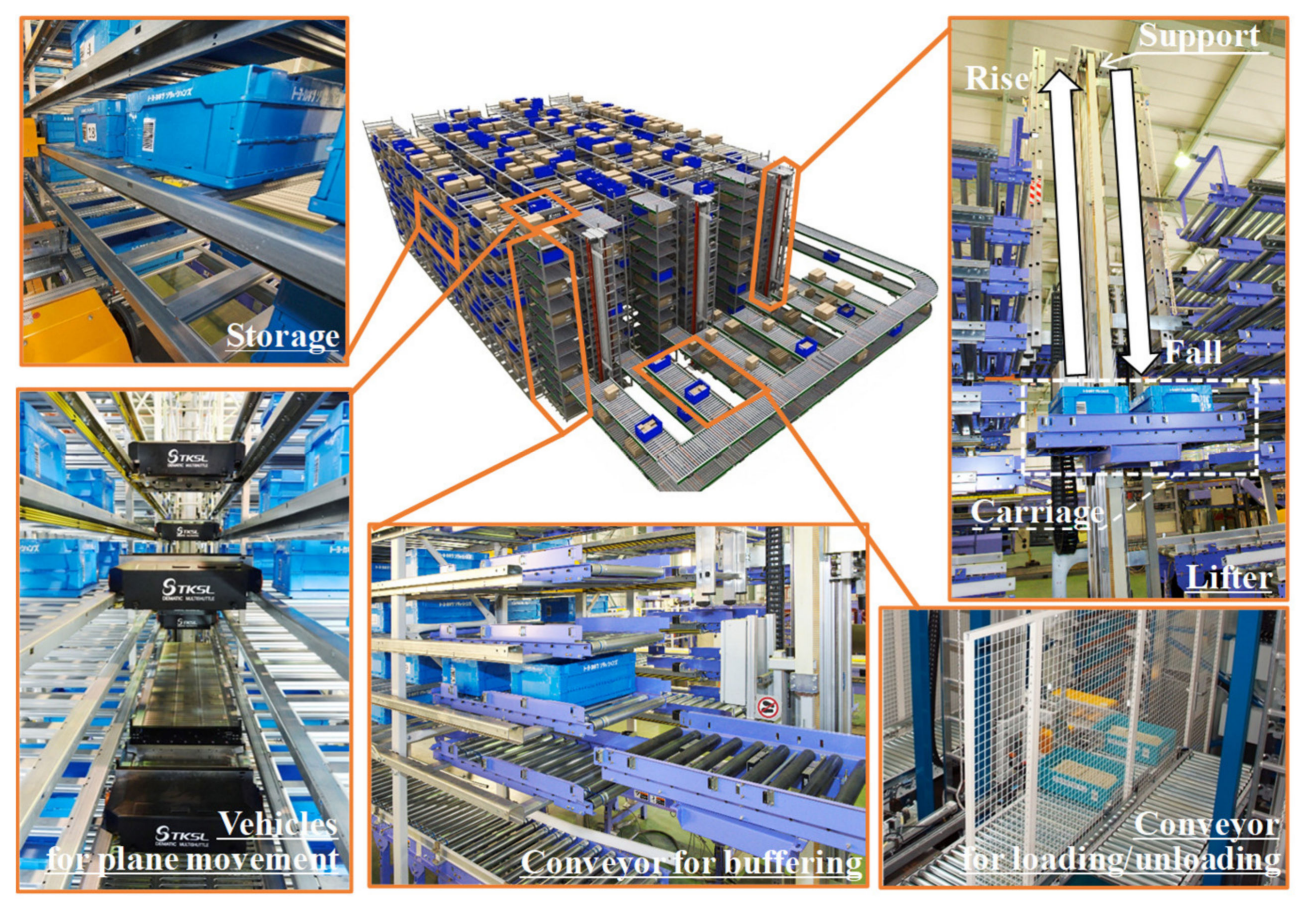

Figure 1. Overview of the entire logistics system. 


\subsection{Calculation of $M D$}

\subsubsection{Normalization of Vibration Data for Creation of Unit Space}

With the proposed method, normal data are used to generate the unit space; this unit space is then used as the criterion to evaluate the characteristic difference between normal and anomalous vibration. Table 1 summarizes the normal data before and after normalization. The physical properties of the lifter gradually deteriorate with the passage of operating time; the vibration characteristics gradually change as a consequence. The operation properties are expressed in terms of the multidimensional $\mathrm{MD}$, and judgment as to whether the operation is proceeding normally or abnormally is validated by using the criterion. As shown in Figure 2a,b, the vibration waveform reflecting the movement of the carriage is acquired by an accelerometer placed in an appropriate position on the body of the lifter. The details of the positioning of the sensors are indicated in Section 4.1. For simplicity, to obtain basic knowledge regarding the effect on the value of MD of damage to each of the various parts, we limited the targeted lifter movement to a single condition: one-way movement from the bottom to the top of the lifter. (We were aware from actual measurement that the influence of the lifter's movement on its vibration characteristics differed slightly depending on whether the movement is rising or descending.) Samples of the waveform in rising were extracted, and their frequency response was obtained using FFT (Fast Fourier Transformation). As shown in Figure 2c,d, the converted results of the frequency characteristic have several peaks. These are caused by the structural resonance of each part of the lifter. It is suggested that the vibration components in the frequency band around the resonance frequencies are largely influenced by aging of the structure. Therefore, in this study, the remarkable peak frequencies that occur during normal operation are read from the spectrum and the energy levels of the signals in the 1/12 octave band range above and below these peak frequencies are calculated. These energy levels are sequentially calculated for each sample waveform generated by each rising. The calculation results for these energy levels are expressed as $x$ in Figure 2c. For example, in the $k$ th operation, the energy levels from $x_{k 1}$ to $x_{k m}$ in $m$ bands are calculated. The unit space of the normal state is generated using the data output from all the energy levels from the first to the $k$ th operation, as shown in Figure 2c. The output data are summarized in section (a-1) of Table 1.

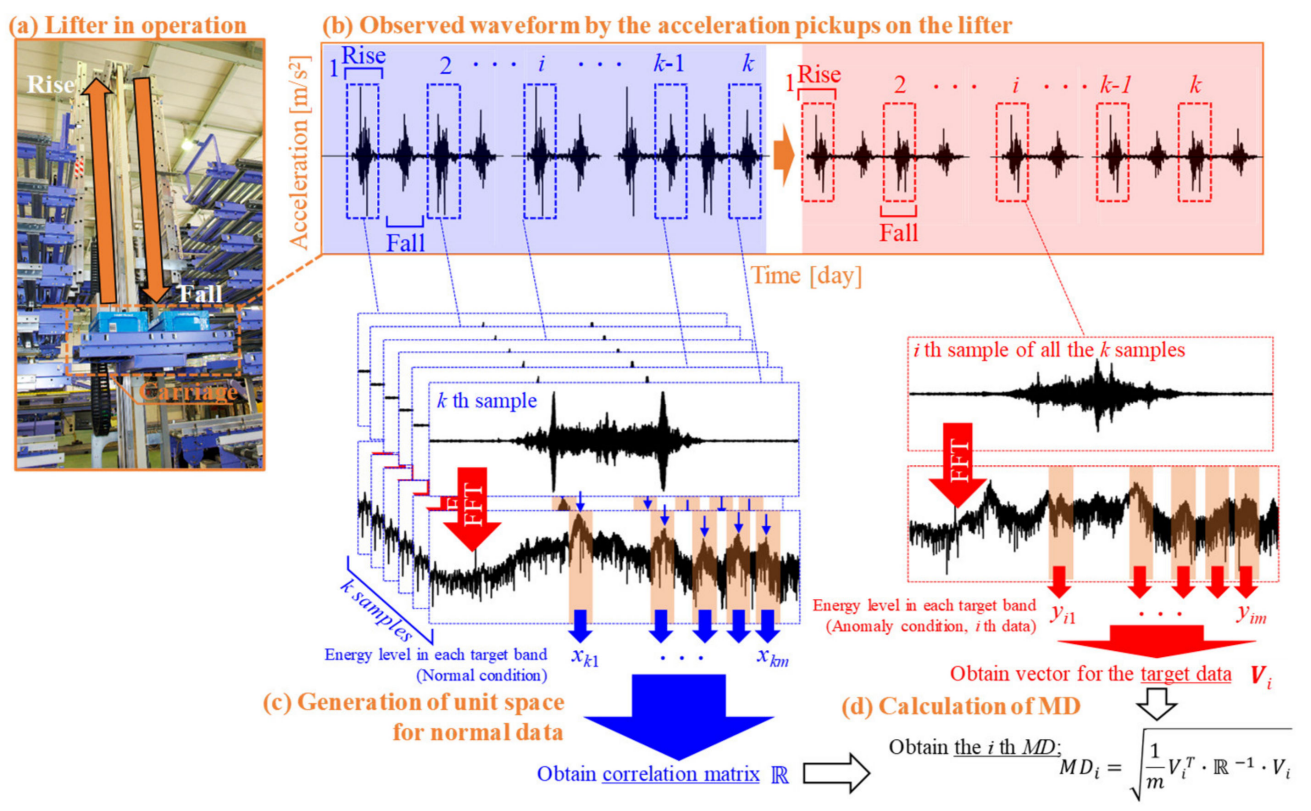

Figure 2. Simulation of Mahalanobis distance (MD) using the vibration acceleration data. The waveforms of the vibration as shown in (b) can be obtained by operating (a) the lifter. Then, by using (c) the generated unit space for normal data, finally (d) the MD can be obtained. 
Table 1. Normalization scheme for the vibration energy levels in each of the frequency bands.

\begin{tabular}{|c|c|c|c|c|c|c|c|c|c|c|c|c|c|}
\hline \multirow{8}{*}{ 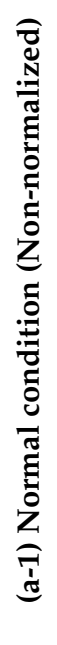 } & & $\begin{array}{l}\text { Band: } \\
\text { No. } 1\end{array}$ & $\cdots$ & $\begin{array}{l}\text { Band: } \\
\text { No. } j\end{array}$ & $\cdots$ & $\begin{array}{l}\text { Band: } \\
\text { No. } m\end{array}$ & \multirow{8}{*}{ 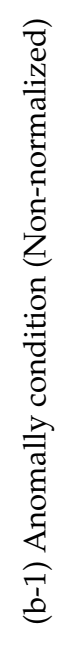 } & & $\begin{array}{l}\text { Band: } \\
\text { No. } 1\end{array}$ & $\cdots$ & $\begin{array}{l}\text { Band: } \\
\text { No. } j\end{array}$ & $\cdots$ & $\begin{array}{l}\text { Band: } \\
\text { No. } m\end{array}$ \\
\hline & Sample: No. 1 & $x_{11}$ & $\cdots$ & $x_{1 j}$ & $\cdots$ & $x_{1 m}$ & & Sample: No. 1 & $y_{11}$ & $\cdots$ & $y_{1 j}$ & $\cdots$ & $y_{1 m}$ \\
\hline & $\cdots$ & $\cdots$ & $\cdots$ & $\cdots$ & $\cdots$ & $\cdots$ & & $\cdots$ & $\cdots$ & $\cdots$ & $\cdots$ & $\cdots$ & $\cdots$ \\
\hline & Sample: No. $i$ & $x_{i 1}$ & $\cdots$ & $x_{i j}$ & $\cdots$ & $x_{i m}$ & & Sample: No. $i$ & $y_{i 1}$ & $\cdots$ & $y_{i j}$ & $\cdots$ & $y_{i m}$ \\
\hline & $\cdots$ & $\cdots$ & $\cdots$ & $\cdots$ & $\cdots$ & $\cdots$ & & $\cdots$ & $\cdots$ & $\cdots$ & $\cdots$ & $\cdots$ & $\cdots$ \\
\hline & Sample: No. $k$ & $x_{k 1}$ & $\cdots$ & $x_{k j}$ & $\cdots$ & $x_{k m}$ & & Sample: No. $k$ & $y_{k 1}$ & $\cdots$ & $y_{k j}$ & $\cdots$ & $y_{k m}$ \\
\hline & Average & $\bar{x}_{1}$ & $\cdots$ & $\bar{x}_{j}$ & $\cdots$ & $\bar{x}_{k}$ & & Average & $\bar{y}_{1}$ & $\cdots$ & $\bar{y}_{j}$ & $\cdots$ & $\bar{y}_{k}$ \\
\hline & $\begin{array}{l}\text { Standard } \\
\text { deviation }\end{array}$ & $\sigma_{1}$ & $\cdots$ & $\sigma_{j}$ & $\cdots$ & $\sigma_{k}$ & & $\begin{array}{l}\text { Standard } \\
\text { deviation }\end{array}$ & $\sigma_{1}$ & $\cdots$ & $\sigma_{j}$ & $\cdots$ & $\sigma_{k}$ \\
\hline \multirow{8}{*}{ 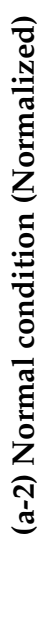 } & & $\begin{array}{l}\text { Band: } \\
\text { No. } 1\end{array}$ & $\cdots$ & $\begin{array}{l}\text { Band: } \\
\text { No. } j\end{array}$ & $\ldots$ & $\begin{array}{l}\text { Band: } \\
\text { No. } m\end{array}$ & \multirow{8}{*}{ 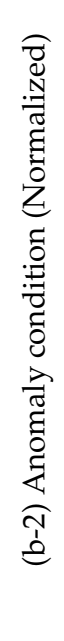 } & & $\begin{array}{l}\text { Band: } \\
\text { No. } 1\end{array}$ & $\cdots$ & $\begin{array}{l}\text { Band: } \\
\text { No. } j\end{array}$ & $\cdots$ & $\begin{array}{l}\text { Band: } \\
\text { No. } m\end{array}$ \\
\hline & Sample: No. 1 & $u_{11}$ & $\cdots$ & $u_{1 j}$ & $\cdots$ & $u_{1 m}$ & & Sample: No. 1 & $v_{11}$ & $\cdots$ & $v_{1 j}$ & $\cdots$ & $v_{1 m}$ \\
\hline & $\cdots$ & $\cdots$ & $\cdots$ & $\cdots$ & $\cdots$ & $\cdots$ & & $\cdots$ & $\cdots$ & $\cdots$ & $\cdots$ & $\cdots$ & $\cdots$ \\
\hline & Sample: No. $i$ & $u_{i 1}$ & $\cdots$ & $u_{i j}$ & $\cdots$ & $u_{i m}$ & & Sample: No. $i$ & $v_{i 1}$ & $\cdots$ & $v_{i j}$ & $\cdots$ & $v_{i m}$ \\
\hline & $\cdots$ & $\cdots$ & $\cdots$ & $\cdots$ & $\cdots$ & $\cdots$ & & $\cdots$ & $\cdots$ & $\cdots$ & $\cdots$ & $\cdots$ & $\cdots$ \\
\hline & Sample: No. $k$ & $u_{k 1}$ & $\cdots$ & $u_{k j}$ & $\cdots$ & $u_{k m}$ & & Sample: No. $k$ & $v_{k 1}$ & $\cdots$ & $v_{k j}$ & $\cdots$ & $v_{k m}$ \\
\hline & Average & $\bar{u}_{1}=0$ & $\cdots$ & $\bar{u}_{j}=0$ & $\cdots$ & $\bar{u}_{m}=0$ & & Average & $\bar{v}_{1}=0$ & $\cdots$ & $\bar{v}_{j}=0$ & $\cdots$ & $\bar{v}_{m}=0$ \\
\hline & $\begin{array}{l}\text { Standard } \\
\text { deviation }\end{array}$ & 1 & $\cdots$ & 1 & $\cdots$ & 1 & & $\begin{array}{l}\text { Standard } \\
\text { deviation }\end{array}$ & 1 & $\cdots$ & 1 & $\cdots$ & 1 \\
\hline
\end{tabular}


To generate the unit space by using the $x_{i j}(1 \leq i \leq k, 1 \leq j \leq m)$ data from $k$ samples (no. 1 to no. $k$ ) with $m$ bands (no. 1 through no. $m$ ), the data are normalized as follows [27]:

$$
u_{i j}=\frac{x_{i j}-\bar{x}_{j}}{\sigma_{j}}
$$

where $\bar{x}_{j}$ and $\sigma_{j}$ indicate the average value and the standard deviation of the $j$ th band, respectively. As a result of normalization, the $x$ data in the (a-1) group in Table 1 are converted to the $u$ data shown in the (a-2) group. Note that $\sigma_{j}$ can be calculated as follows:

$$
\sigma_{j}=\sqrt{\frac{1}{k} \sum_{i=1}^{k}\left\{x_{i j}-\bar{x}_{j}\right\}^{2}}
$$

As shown in section (a-2) of Table 1 , the normalized data indicate $\bar{x}_{j}=1.0$ and $\sigma_{j}=0.0$.

\subsubsection{Generation of Correlation Matrix}

The correlation coefficient measuring the correlation between $u_{i j_{1}}(1 \leq i \leq k)$ in the $j_{1}$ th band and $u_{i j_{2}}(1 \leq i \leq k)$ in the $j_{2}$ th band is calculated as

$$
r_{j_{1} j_{2}}=\frac{\sum_{i=1}^{k}\left(u_{i j_{1}}-\bar{u}_{j_{1}}\right) \cdot\left(u_{i j_{2}}-\bar{u}_{j_{2}}\right)}{\sqrt{\sum_{i=1}^{k}\left(u_{i j_{1}}-\bar{u}_{j_{1}}\right)^{2} \cdot \sum_{i=1}^{k}\left(u_{i j_{2}}-\bar{u}_{j_{2}}\right)^{2}}}
$$

Since $u$ has already been normalized, the relationships

$$
\begin{gathered}
\sum_{i=1}^{k}\left(u_{i j_{1}}-\bar{u}_{j_{1}}\right)^{2}=k \\
\sum_{i=1}^{k}\left(u_{i j_{2}}-\bar{u}_{j_{2}}\right)^{2}=k \\
\bar{u}_{j_{1}}=0 \\
\bar{u}_{j_{2}}=0
\end{gathered}
$$

are satisfied and $r$ can be calculated as

$$
r_{j_{1} j_{2}}=\frac{\sum_{i=1}^{k} u_{i j_{1}} \cdot u_{i j_{2}}}{k}
$$

Finally, the correlation matrix $\mathbb{R}$ can be shown as

$$
\mathbb{R}=\left[\begin{array}{cccccc}
1 & r_{12} & \cdots & r_{1 j_{2}} & \cdots & r_{1 m} \\
r_{21} & 1 & \cdots & r_{2 j_{2}} & \cdots & r_{2 m} \\
\vdots & \vdots & \vdots & \vdots & \vdots & \vdots \\
r_{j_{1} 1} & r_{j_{1} 2} & \cdots & 1 & \cdots & r_{j_{1} m} \\
\vdots & \vdots & \vdots & \vdots & \vdots & \vdots \\
r_{k 1} & r_{k 2} & \cdots & r_{k j_{2}} & \cdots & 1
\end{array}\right]
$$

The correlation matrix $\mathbb{R}$ will be used in the next section to obtain MD. 


\subsubsection{Calculation of MD}

Normalization is performed on data group $y$ (Table $1(\mathbf{b}-1)$ ), the group to be judged as either normal or anomalous, to produce data group $v$ (Table $1(\mathrm{~b}-2)$ ). Note that the normalization here is not based on the average and standard deviation of each band of data group $y$, but rather, it is based on the average and standard deviation of each band of data group $x$. First, the following column vector $V_{i}$ is composed by using the $i$ th data value $v_{i j}(1 \leq j \leq m)$ :

$$
V_{i}=\left[\begin{array}{c}
v_{i 1} \\
v_{i 2} \\
\vdots \\
v_{i j} \\
\vdots \\
v_{i m}
\end{array}\right]
$$

The value of MD for the $j$ th sample can then be calculated as [17]

$$
\mathrm{MD}_{v_{j}}=\sqrt{\frac{1}{k}\left[v_{i 1} \cdots v_{i m}\right] \cdot \mathbb{R}^{-1} \cdot\left[\begin{array}{c}
v_{i 1} \\
\vdots \\
v_{i m}
\end{array}\right]}
$$

Herein, the value of MD indicates the physical distance between the mean value of the unit space created by the normal data group $x$ and the judged data of the group $y$. Therefore, as the MD is larger, the frequency characteristics of the judged data $y$ is more different from the normal data group $x$. Therefore, the value of MD is used as the indicator of the abnormality of the mechanical system in this study.

\subsection{Quantitative Evaluation of $M D$}

If each feature value for the normalized data used to generate the unit space follows a normal distribution, then $k \cdot \mathbf{M D}_{v_{j}}{ }^{2}$ follows a $\chi^{2}$ distribution with $k$ degrees of freedom [16,17]. In this study, the number of bands is $k=4$, as will be described later. To confirm the statistical properties such as the normality of the data, Figure 3 shows the probability density distribution of the calculation results of $k \cdot \mathbf{M D}_{v_{j}}{ }^{2}$ in the normal case. Here, it can be seen that $k \cdot \mathbf{M D}_{v_{j}}{ }^{2}$ follows the $\chi^{2}$ distribution with four degrees of freedom. The $\chi^{2}$ distribution with four degrees of freedom has a critical value of 13.3 when the probability level is set at 0.01 . Therefore, in this study, the evaluated vibration is regarded as anomalous when $\mathbf{M D}_{v_{j}}{ }^{2}$ exceeds $13.3 / 4=3.32$. In order to make it easier to refer to this level, the threshold value for an anomaly was set to $\mathbf{M D}^{2}$, obtained by simply squaring MD.

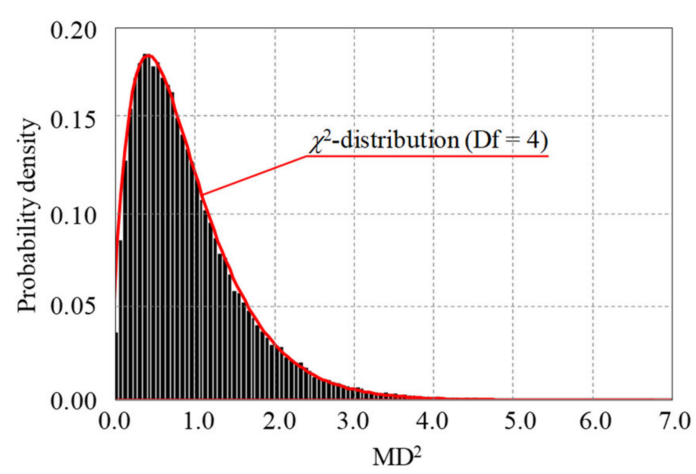

Figure 3. Probability density distribution of calculated results when operating in the normal condition. 


\section{Basic Study for Calculation Scheme of MD}

The purpose of this study is to determine the mechanical characteristics of the lifter operation. However, to use the MT method for the vibration waveform of the various parts of the lifter, which has a complex assembly structure, it was first necessary to confirm the applicability of the MT method in the case of a simple mechanical device. In this study, it was found that the vibration characteristics measured at each part of the lifter have several peaky frequency components that correspond to the modal components of the lifter. Therefore, a basic study was performed using a shaker (Figure 4) that was excited by a signal superimposed with pure tones at ten frequencies. The time waveform of the output vibration was then measured, and the basic validity of the method to obtain $\mathrm{MD}^{2}$ was verified. The procedure is described in greater detail below.

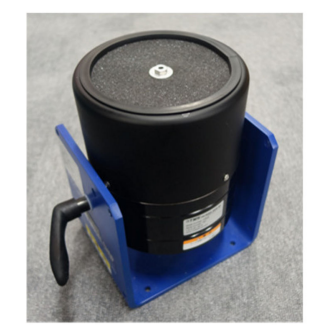

Figure 4. Adopted shaker in the basic investigation.

First, a steady-state signal with the frequency characteristics shown in Figure 5a was generated by superimposing pure tones at every $200 \mathrm{~Hz}$ in the frequency range from $200 \mathrm{to} 2.2 \mathrm{kHz}$. The shaker was excited by this signal, and the acceleration waveforms of the shaker were continuously acquired over three days. The measured continuous waveforms were cut out every $6.5 \mathrm{~s}$ to generate 13,300 data fragments per day for a total of 39,900. Then, the unit space was generated using the data obtained on the first day and the $\mathrm{MD}^{2}$ for the waveforms over the entire three days was calculated by using the unit space. While, as described here, the vibration frequency components of each frequency band are used to calculate the value of $\mathrm{MD}^{2}$, the scheme for determining the bandwidth to efficiently represent the frequency feature of vibration is discussed next through case studies based on two types of selection methods.

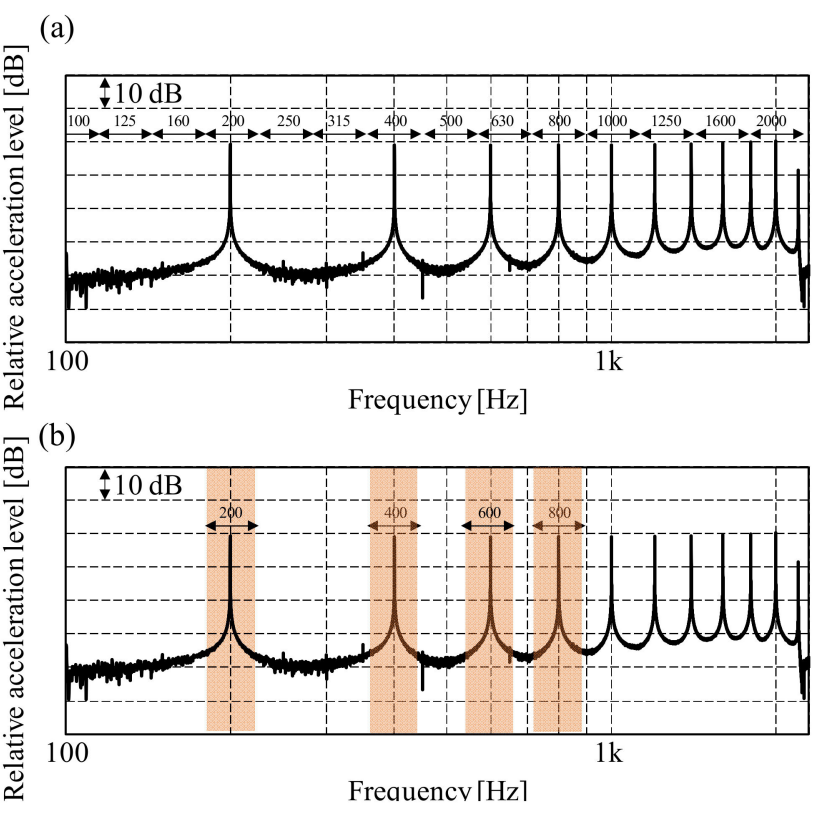

Figure 5. Two methods for selecting frequency bands: (a) using a constant percentage bandwidth and (b) using peak frequencies. 


\section{1. $M D^{2}$ Calculation Using Frequency Components in Constant Percentage Bandwidth}

We sought to calculate $\mathrm{MD}^{2}$ by first calculating the energy level in each of the 14 constant percentage bandwidths in the $1 / 3$ octave band from 100 to $2 \mathrm{kHz}$; the value of $\mathrm{MD}^{2}$ could then be calculated by using the 14 energy levels as the band information for $k=14$ in the MT method (Figure 5a). Figure 6a shows the fluctuation of the energy level in each band over the three-day period. While the measurements were recorded under ideal state conditions with little disturbance vibration, slight fluctuations can be observed, particularly in the $200 \mathrm{~Hz}$ band. Figure 7 shows the $\mathrm{MD}^{2}$ calculation results based on the data in Figure 6a. The average $\mathrm{MD}^{2}$ for the fragment data between 1 and 13,300 on the first day is approximately 1.0. This seems natural, since the data that generated the unit space are evaluated in the unit space. On the other hand, even though the vibration was continuously output in the steady state, the $\mathrm{MD}^{2}$ values after the second day greatly exceeded 1.0, and a stable $\mathrm{MD}^{2}$ result was not obtained despite the vibration being essentially in steady state. This caused us to speculate that the calculated values of $\mathrm{MD}^{2}$ are materially affected by minute time fluctuations in vibration.

(a)

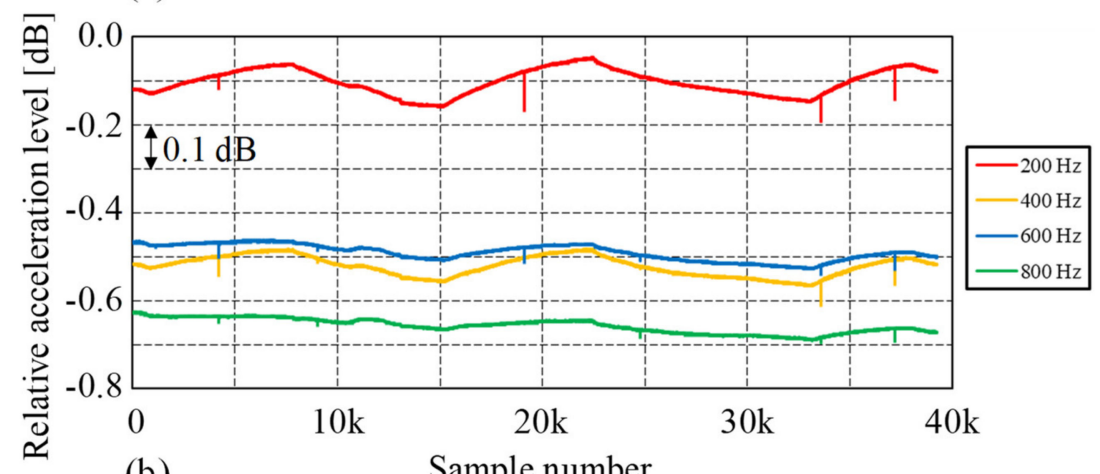

(b)

Sample number

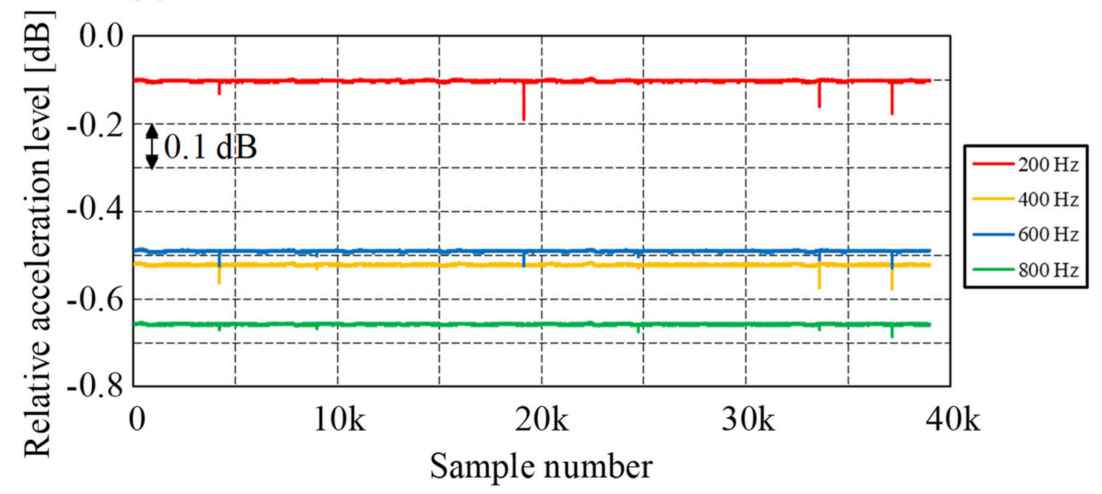

Figure 6. (a) Time-series data of the energy levels in each frequency band and (b) those treated with high-pass filtering.

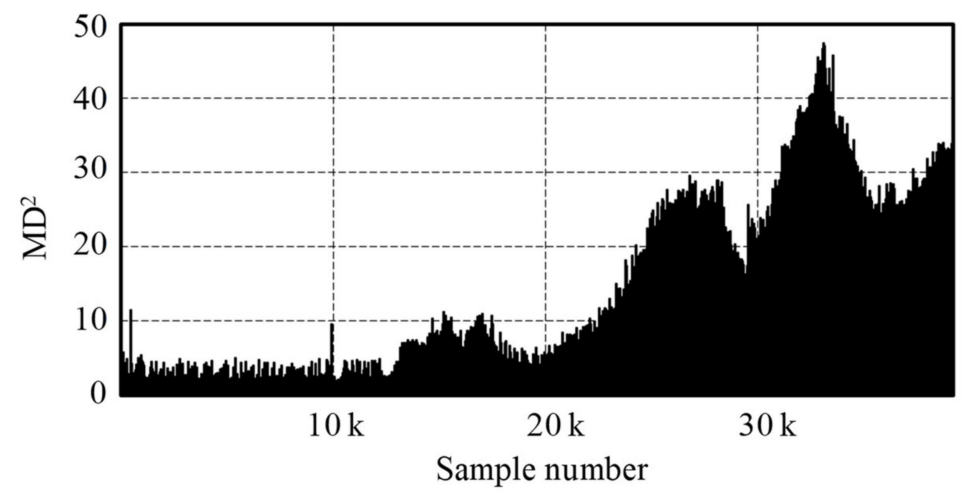

Figure 7. $\mathrm{MD}^{2}$ values using constant percentage bandwidth in the $1 / 3$ octave band. 


\subsection{Calculation Using Frequency Components at Around Peak Frequencies}

As noted, when the method described in Section 3.1 was used, the $\mathrm{MD}^{2}$ values increased even though the equipment was operating in a steady-state condition. To cope with this problem, an alternative method was considered. Specifically, as shown in Figure 5b, the frequency components used to calculate $\mathrm{MD}^{2}$ were extracted as 1/12 octave bands around the peaks of the frequency characteristics at $200 \mathrm{~Hz}, 400 \mathrm{~Hz}, 600 \mathrm{~Hz}$, and $800 \mathrm{~Hz}$. The reason for this is that background noise components other than peak frequency are included in the data when the energy levels in all of the $1 / 3$ octave bands are used; however, these background noise data are not related to equipment malfunction. Therefore, developing a method that uses only the data around the peak frequencies would seem appropriate.

In addition to the abovementioned treatment, the minute fluctuations in vibration observed in Figure 6a may affect the increase in the value of $\mathrm{MD}^{2}$. To establish the periodicity of these fluctuations, the results of FFT processing at the sampling frequency of 13,300 Hz in the $200 \mathrm{~Hz}$ band with the largest fluctuation among all the bands in Figure 8 was used as a reference. It was observed that the periodic components in the low-frequency range were dominant. For this reason, a high-pass filter with a cutoff frequency of $4.16 \mathrm{~Hz}$ in the sampling frequency of $13,300 \mathrm{~Hz}$ was applied to the time-series data of the energy level in each band, and the effects of the fluctuations were cut off to the extent possible. Note that the cycle in $4.16 \mathrm{~Hz}$ corresponds to periodic components over approximately four hours. The filtered time-series data are shown in Figure $6 \mathrm{~b}$. It can be seen that the minute time fluctuations have been eliminated. Figure 9 shows the calculated $\mathrm{MD}^{2}$ results. The $\mathrm{MD}^{2}$ values from the 1 to 13,300 time-series data on the first day have an average of approximately 1.0; the average after the second day is approximately 1.0 as well. Thus, a relatively constant $\mathrm{MD}^{2}$ is observed.

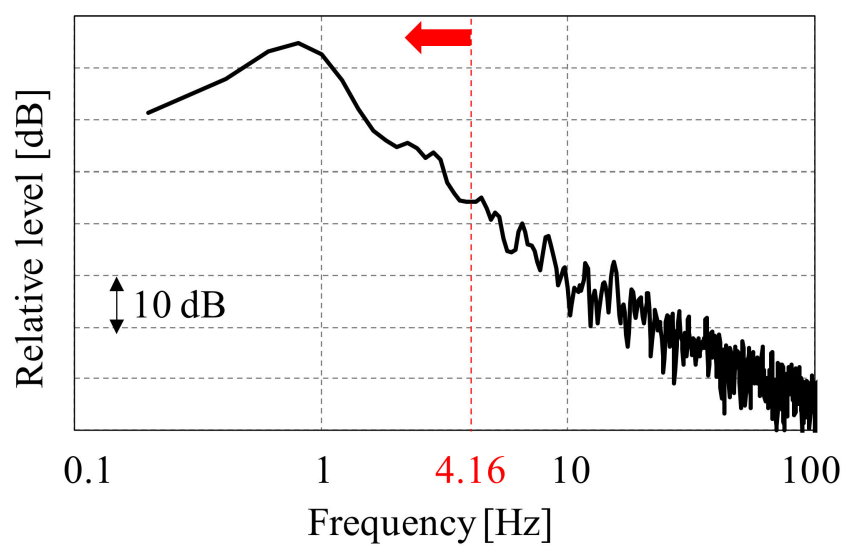

Figure 8. Frequency components of the fluctuation in the vibration time-series data for the lifter under normal condition.

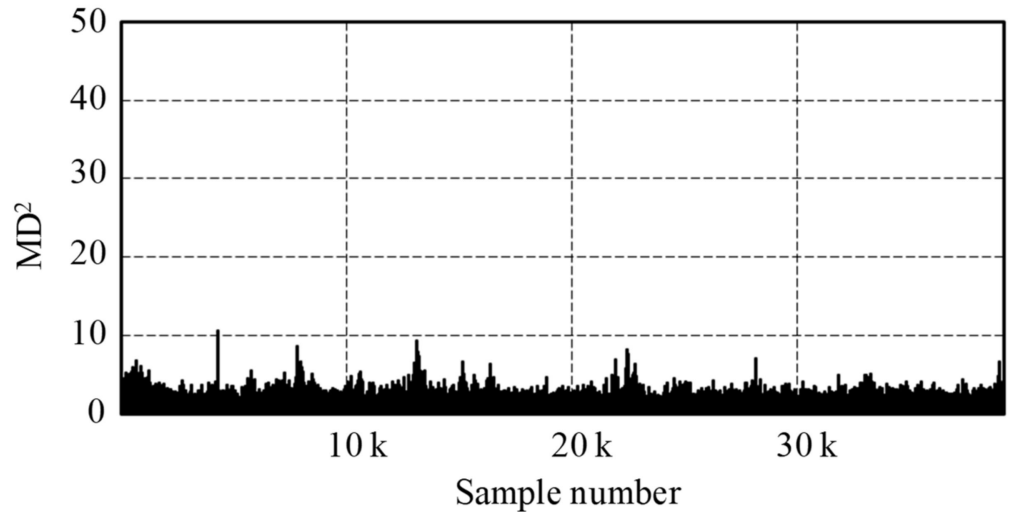

Figure 9. $\mathrm{MD}^{2}$ calculated based on the optimal selection of the frequency band around each of the peak frequencies. 
From the results in Sections 3.1 and 3.2, when the MT method is applied to a vibration waveform having peak components, relatively stable $\mathrm{MD}^{2}$ values of approximately 1.0 during normal operation are obtained by using the energy level in the frequency band around the peak components of the target waveform, making this the preferred alternative. Moreover, a periodic fluctuation component longer than approximately six hours should be cut off.

\section{Validation of MD Calculation Scheme for Lifter Operation}

The validity of the method described in Section 3 for detecting the failure of the lifter operation was subsequently tested. To verify the change in vibration characteristics caused by mechanical failure, three kinds of damage were artificially inflicted on parts of the lifter, and their effects on the $\mathrm{MD}^{2}$ results were examined.

\subsection{Calculation Using Frequency Components Around Peak Frequencies}

The vibration characteristics of the lifter were carefully determined. Figure 10 shows in detail the configuration of the target lifter. The lower left of panel Figure 10a shows a pair of assembled lifters, only one of which was operated and targeted in this study. The lifter has a carriage (Figure 10c) for loading products. The lifter has a belt to move the carriage up and down and a pulley to operate the belt. The pulleys are located at the bottom and top of the lifter, shown in Figure 10b,d, respectively. To monitor the vibration of the lifter, two accelerometers, R1 and R2 (PCB, 603C01, upper limit frequency: $10 \mathrm{kHz}$ within a variance of $\pm 3 \mathrm{~dB}$ ), were set at the positions shown in Figure 11 . As will be described later, the receiver at R1 was set to determine the mechanical conditions of the belt and guide wheel, whereas that at $\mathrm{R} 2$ was set to determine that of the bearings. The reason for the location of these sensors are as follows: Firstly, the vibration caused by the contact between the pulley and belt shown in the right figure of Figure 11a can be sensitively captured at the receiver R1. In addition, the vibration caused by the contact between the guide wheel on the carriage (Figure 10c) and the aluminum column easily transmits via the aluminum column and can be sensitively captured at receiver R1 as well. On the other hand, since the rotational vibration caused by the bearing is relatively weak compared to the vibration caused by the above devices that needs capturing on the surface of the bearing shown in the right figure of Figure 11b, each was attached magnetically to a steel plate. Receiver R1 was set on the steel plate surrounding the pulley, while R2 was set on the steel plate near the bearings used when the pulley at the top of the lifter is rotated. Each of the lifter parts-the belt (Figure 12A), the guide wheel (Figure 12B), and the bearings (Figure 12C)—was artificially damaged. For the belt, the green coating material on the side meshing with the pulley was sanded to make the meshing of the pulley and the belt unstable. For the guide wheel, its rubber-coated surface was melted, making the carriage operation unstable. Finally, a vertical force totaling $1 \mathrm{kN}$ was applied to each of the bearings to destabilize the pulley rotation. 


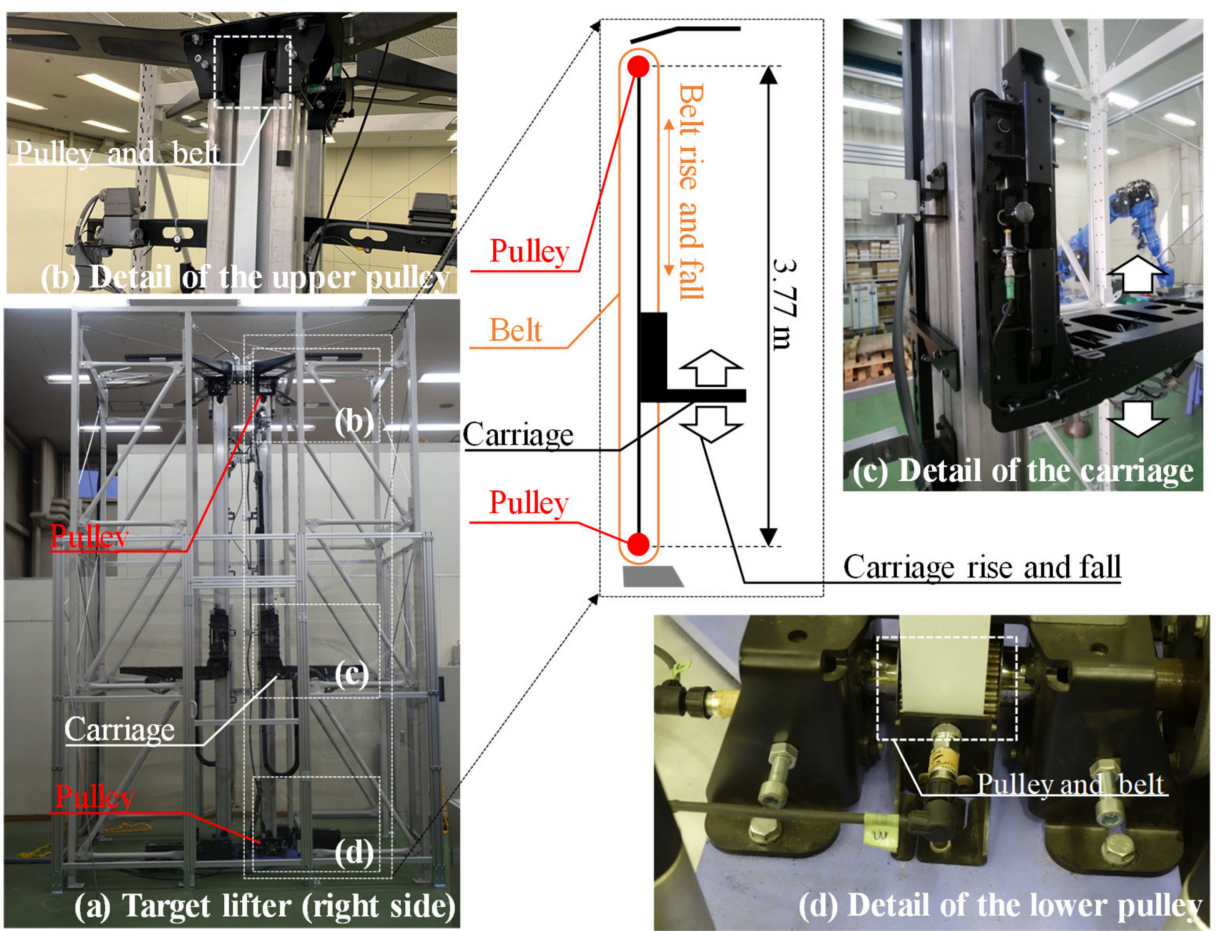

Figure 10. Detailed configuration of the target lifter. In this figure, (a) target lifter of the right side, details of the (b) upper pulley, (c) carriage, and (d) lower pulley are indicated.

(a) Receiving point of $\mathrm{R} 1$ at the lower part of the lifter

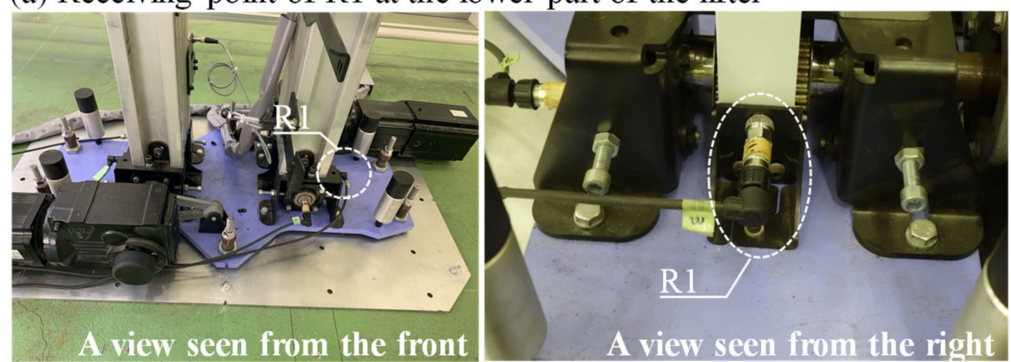

(b) Receiving point of R2 at the upper part of the lifter

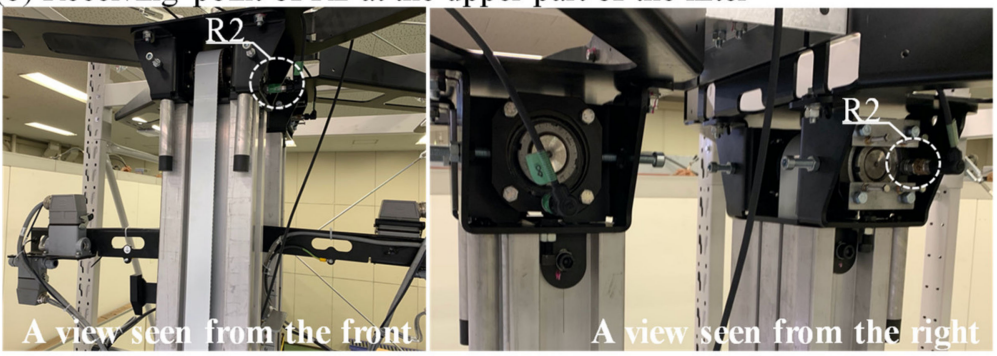

Figure 11. Details of the setting of the vibration receivers on the surface of the lifter. In these pictures, the upper one indicates (a) receiving point of R1, while the lower one indicates (b) that of R2. 
(A) Damaged part on the surface of the belt

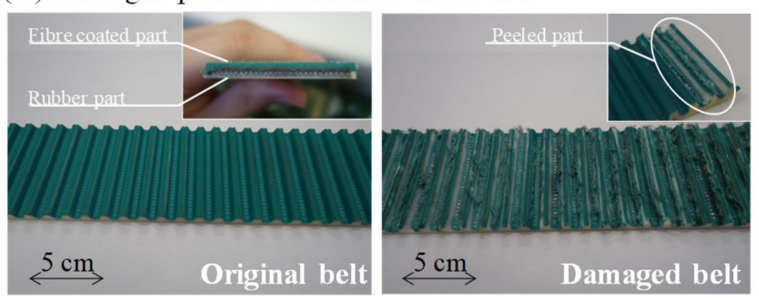

(B) Damaged part on the wheel

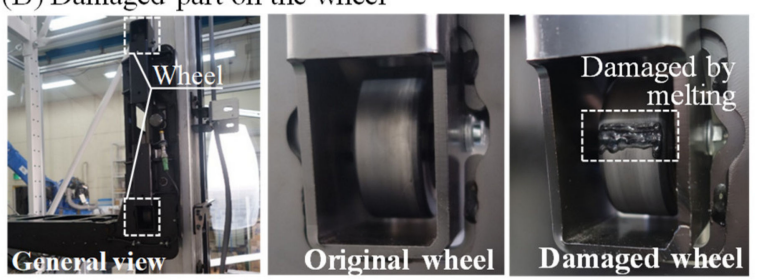

(C) Loading on each of the bearing

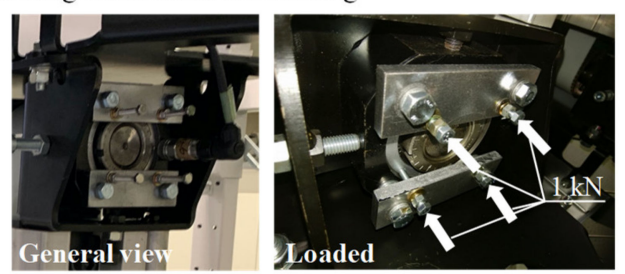

Figure 12. Artificially damaged conditions: (A) damage to belt, (B) damage to guide wheel, and (C) damage to bearings.

\subsection{Mechanical Effect of Artificial Damage on Vibration Characteristics of Lifter}

The acceleration waveforms of the unloaded lifter under three different conditions are shown in Figure 13: operating in the normal state (Figure 13(a1)), operating with a damaged belt

(Figure 13(a2)), and operating with a damaged guide wheel (Figure 13(a3)). All of these results were measured by receiver R1. In this study, the lifter carriage continuously moved up and down in the range between 0.3 and $2.8 \mathrm{~m}$ above the ground. Each round trip of the carriage took $8.6 \mathrm{~s}$. Thus, the carriage made 418 trips per hour or 10,046 trips per day. As can be seen in Figure 14, the vibration waveforms during the rising and descending phases of operation are slightly different. In this study, only the vibration waveform for the rising phase was used. Looking at the waveforms shown in Figure 13(a1)-(a3), it can be seen that they are quite different depending on the type of damage.

The spectrograms obtained from the time waveforms are shown in Figure 13(b1)-(b3), respectively. When the carriage rises, it first starts to move, then accelerates, reaches maximum speed, then decelerates, and finally stops at the top. As can be seen from these spectrograms, the eminent frequency of the main frequency component of vibration begins to rise at the start of operation, then begins to fall as deceleration starts. Although the spectrogram of the respective conditions has slightly different features, it is difficult to determine the effect of each type of damage on the time-frequency response. Consequently, the results of FFT processing of the waveforms in Figure 13(a1)-(a3) are shown in Figure 15. As can be seen in Figure 15a, the frequency characteristics of the peaks and dips when operating in the normal condition differ from those associated with operating with a damaged belt. Figure $15 \mathrm{~b}$ also shows some differences between operating in the normal condition and operating with a damaged guide wheel. 
(a1) Normal condition

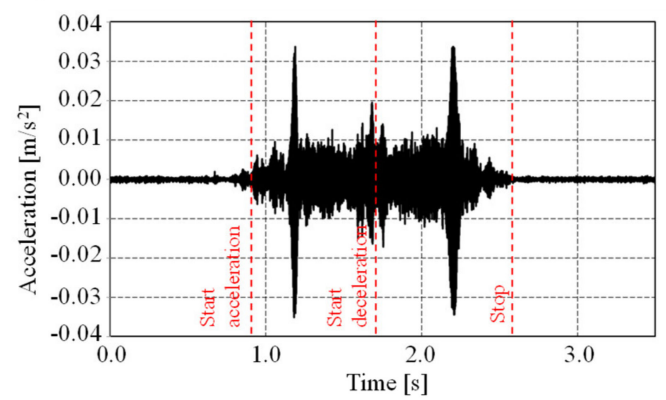

(a2) Condition with damage on the belt

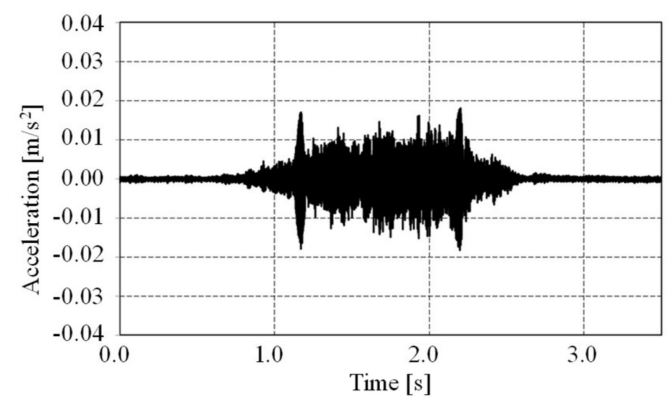

(a3) Condition with damage on the wheel

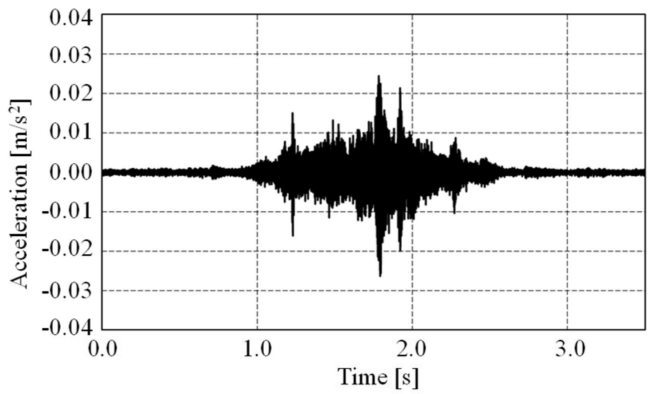

(b1) Normal condition

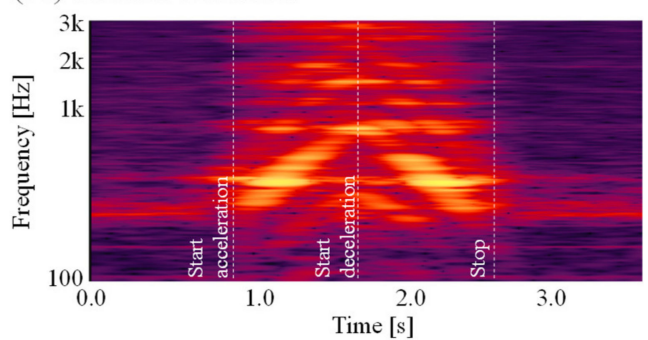

(b2) Condition with damage on the belt



(b3) Condition with damage on the wheel

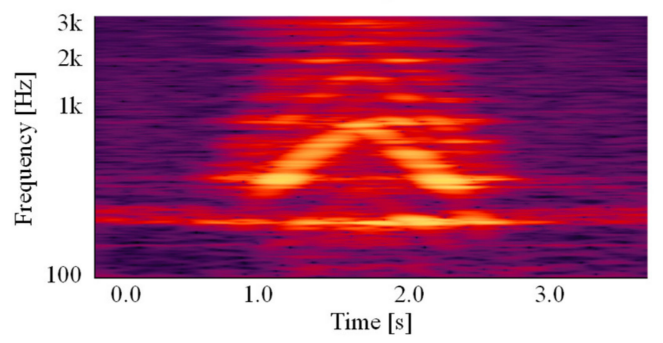

Figure 13. Acceleration waveforms and their spectrograms for the lifter $(\mathbf{a} 1, \mathbf{b} 1)$ operating in normal state, $(\mathbf{a} 2, \mathbf{b} 2)$ operating with a damaged belt, and $(\mathbf{a} 3, \mathbf{b} 3)$ operating with a damaged guide wheel. As shown in Figure 13(a1) and (b1), the waveform starts to sweep up after around $0.9 \mathrm{~s}$ when the lifter starts to accelerate; after that, the waveform starts to sweep down after $1.8 \mathrm{~s}$; then finally, the large fluctuation of the waveform finishes at around $2.8 \mathrm{~s}$ when the lifter stops.

Figure 16 shows the time waveforms and spectrograms obtained by R2 under the normal condition and under the condition of damaged (impeded) bearings. The differences between the normal and damaged conditions observed in the time waveform and spectrogram are relatively small compared to those shown in Figure 13. Figure 17 shows the frequency characteristics in these conditions. In these results, the characteristic difference in the peak-dip tendencies shown in Figure 15 is not observed; however, in the damaged-bearing condition, the energy level is approximately $5 \mathrm{~dB}$ lower than under the normal condition over the entire frequency range. 


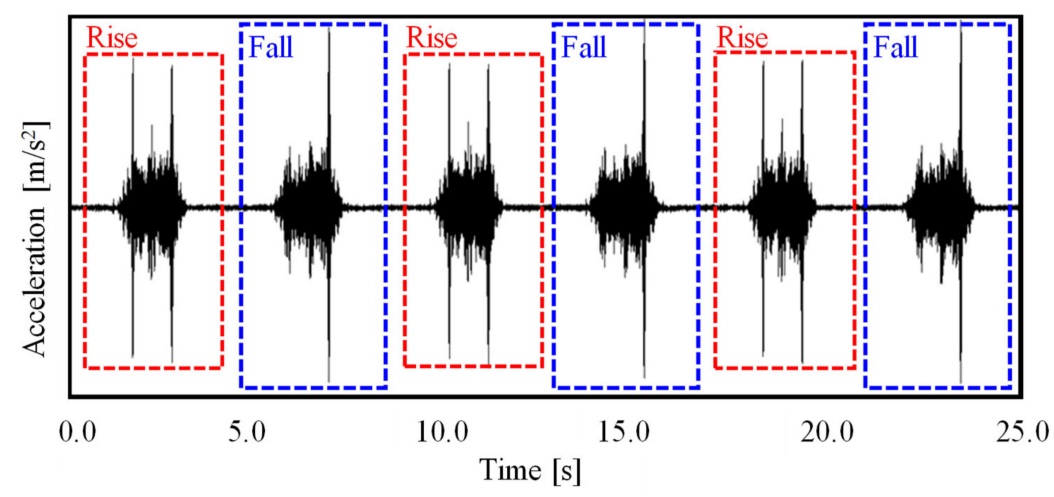

Figure 14. Example of the acceleration waveform for the lifter when rising and descending: These vibration waveforms during the rising and descending phases of operation are slightly different. In this study, only the vibration waveform for the rising phase was used.
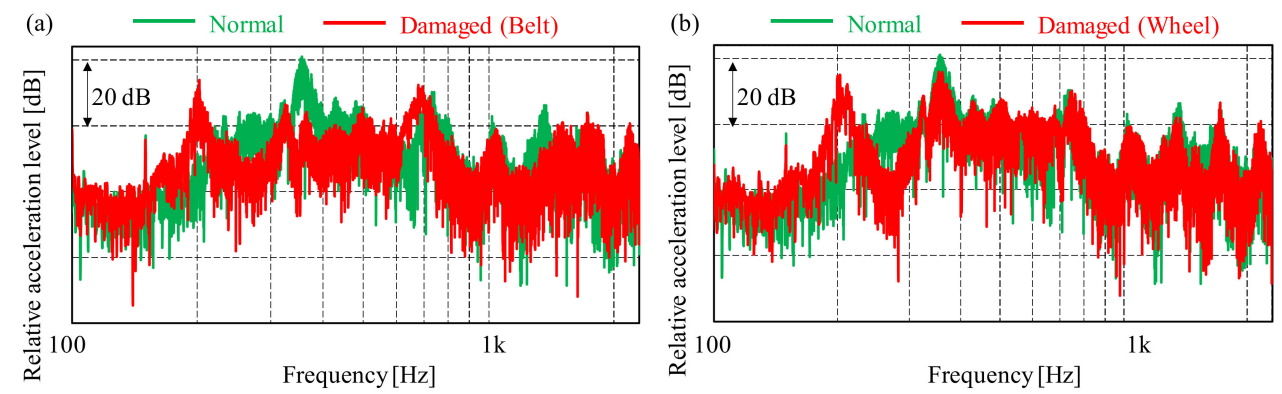

Figure 15. Comparison of the frequency characteristics of the vibration in the normal condition and each of two damage conditions: damaged belt and damaged guide wheel. (a) The frequency characteristics of the peaks and dips when operating in the normal condition differ from those associated with operating with a damaged belt. (b) Shown are some differences between operating in the normal condition and operating with a damaged guide wheel.
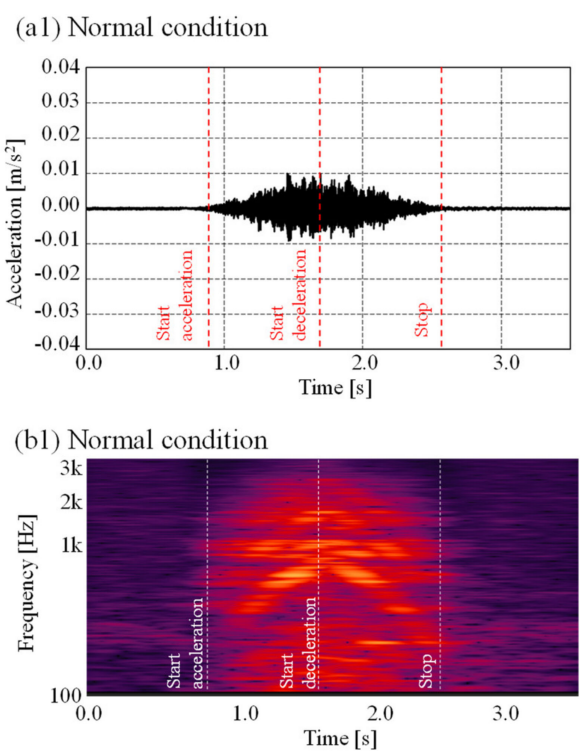

(a2) Condition with damage on the bearing

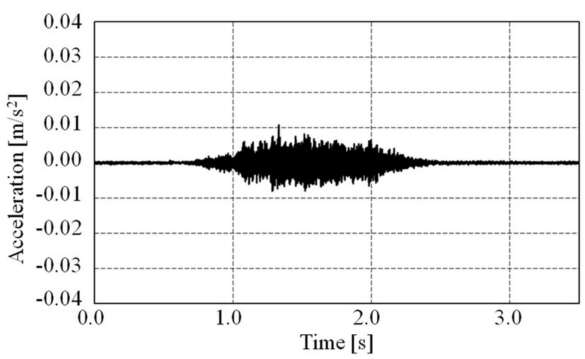

(b2) Condition with damage on the bearing

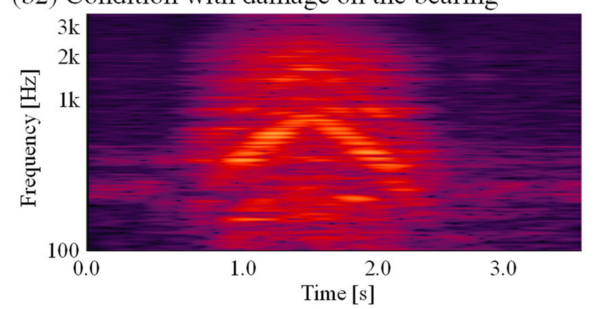

Figure 16. Acceleration waveforms and their spectrograms for the lifter $(\mathbf{a 1}, \mathbf{b} 1)$ in the normal state and $(\mathbf{a 2}, \mathbf{b 2})$ operating with a damaged bearing: As shown in Figure 16(a1) and (b1), the waveform starts to sweep up after around $0.9 \mathrm{~s}$ when the lifter starts to accelerate; after that, the waveform starts to sweep down after $1.8 \mathrm{~s}$; then finally, the large fluctuation of the waveform finishes at around $2.8 \mathrm{~s}$ when the lifter stops. The differences between the normal and damaged conditions observed in the time waveform and spectrogram are relatively small compared to those shown in Figure 13. 


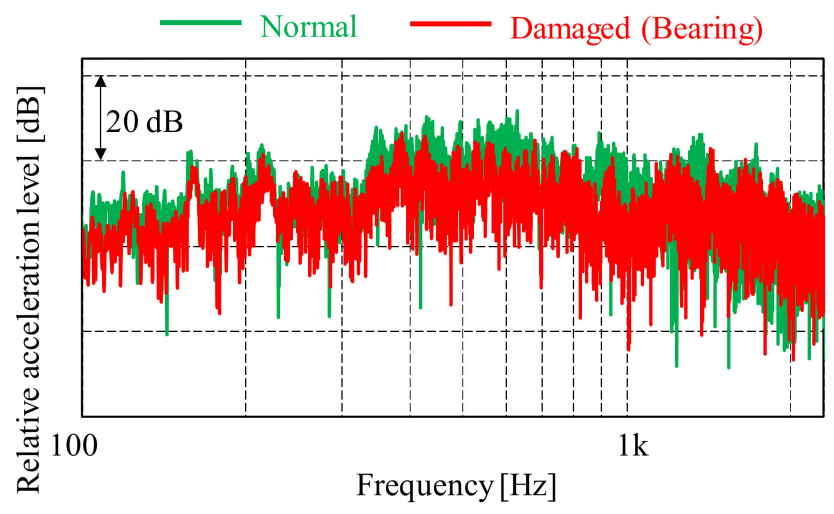

Figure 17. Comparison of the frequency characteristics of the vibration in the normal condition and when operating with a damaged bearing: This result shows that the characteristic difference in the peak-dip tendencies received at R1 shown in Figure 15 is not observed; however, in the damaged-bearing condition, the energy level is approximately $5 \mathrm{~dB}$ lower than under the normal condition over the entire frequency range.

The above results suggest that differences in the mechanical state of the equipment from the normal state can be detected from the vibration characteristics under each of the three conditions tested: a damaged belt, a damaged guide wheel, and a damaged bearing.

\subsection{Effect of Each Type of Damage on $M D^{2}$}

\subsubsection{Calculation of $\mathrm{MD}^{2}$ Under Normal Condition}

To calculate $\mathrm{MD}^{2}$ under the normal condition, the unloaded lifter was continuously operated in the normal state for nine days. A unit space was generated based on the fragment data (10,046 waveforms) obtained from measurements on the first day; the $\mathrm{MD}^{2}$ of the fragment data for the remaining eight days $(80,368$ waveforms) in the generated unit space was then produced using the method described in Section 2.

From the frequency characteristics of the vibration data obtained from R1 during normal operation (Figure 18), the energy levels in the 1/12 octave band around the four prominent peak frequencies at $724 \mathrm{~Hz}, 1020 \mathrm{~Hz}, 1378 \mathrm{~Hz}$, and $1744 \mathrm{~Hz}$ were obtained and used for the calculation of MD2. Note that the frequency components around the lowest peak at $340 \mathrm{~Hz}$ were excluded from the calculation since the level fluctuation in each operation was quite large. Figure 19 shows the time series of the energy levels in each of the four selected frequency bands. Since some trends in the time series of the energy levels were apparent, the periodic components of these series were checked using the results of FFT processing at the sampling frequency of 10,046 Hz. The frequency component of $724 \mathrm{~Hz}$ that included the largest trend component is shown in Figure 20. Since long-term periodic components were dominant in the quite low-frequency range, a high-pass filter with a cutoff frequency of $4.16 \mathrm{~Hz}$ in the sampling frequency of 10,046 $\mathrm{Hz}$ was applied to the time-series data in each band and the effects of the fluctuations were cut off. The filtered time-series data of the energy levels and the $\mathrm{MD}^{2}$ values obtained from these data are shown in Figures $19 \mathrm{~b}$ and 21, respectively. The trends were removed by the filtering, and the $\mathrm{MD}^{2}$ values during the nine days of normal operation appear to be relatively constant around an average value of 0.94 . 


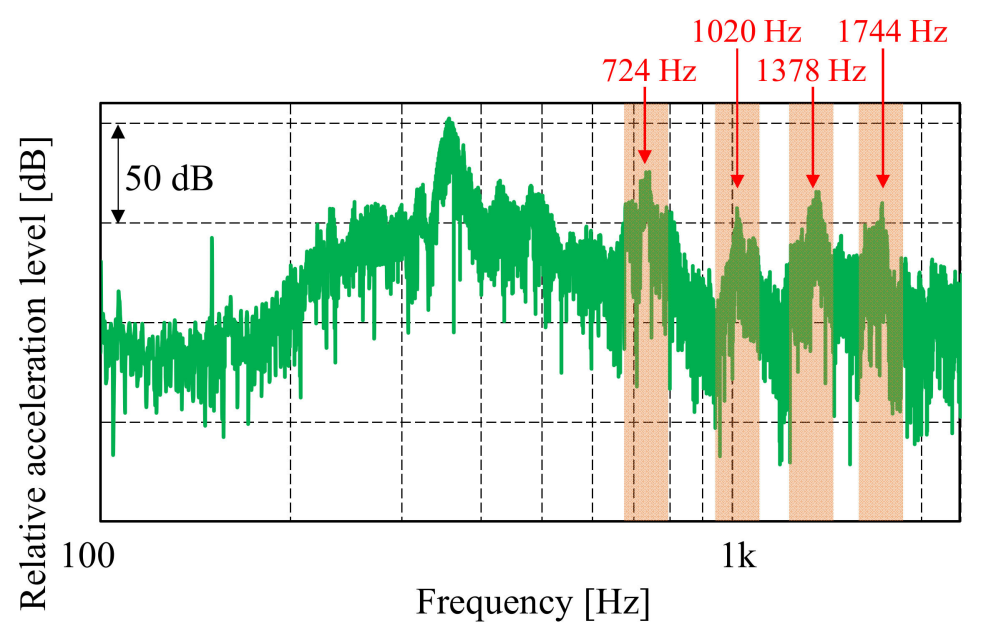

Figure 18. Selection method using frequency bands to calculate the $\mathrm{MD}^{2}$ of the lifter: From these frequency characteristics of the vibration data obtained from R1 during normal operation, the energy levels in the $1 / 12$ octave band around the four prominent peak frequencies at $724 \mathrm{~Hz}, 1020 \mathrm{~Hz}, 1378 \mathrm{~Hz}$, and $1744 \mathrm{~Hz}$ were obtained and used for the calculation of $\mathrm{MD}^{2}$.

(a)

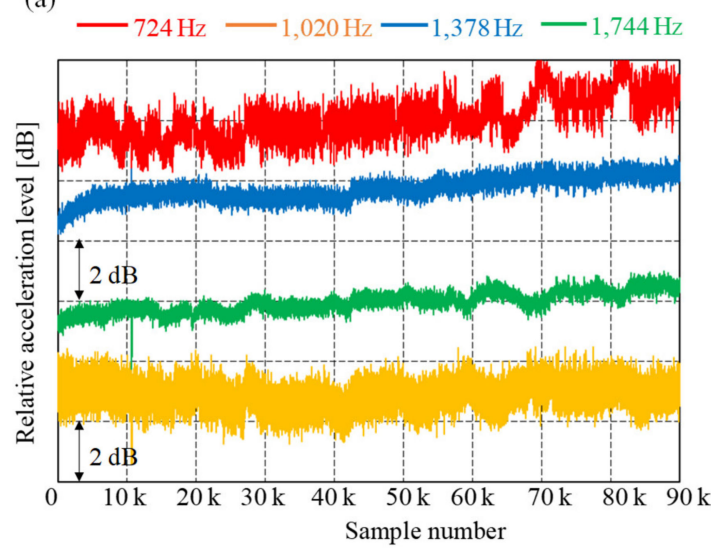

(b)

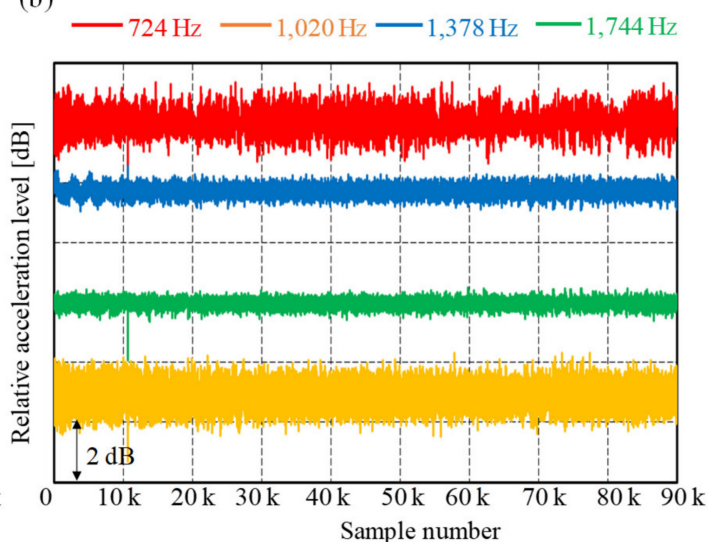

Figure 19. Time-series data of the vibration in the normal conditions (a) without high-pass filtering and (b) with high-pass filtering: Since some trends in the time series of these energy levels in the respective bands were apparent, the periodic components of these series were investigated using the results of FFT processing, and will be removed by filtering.

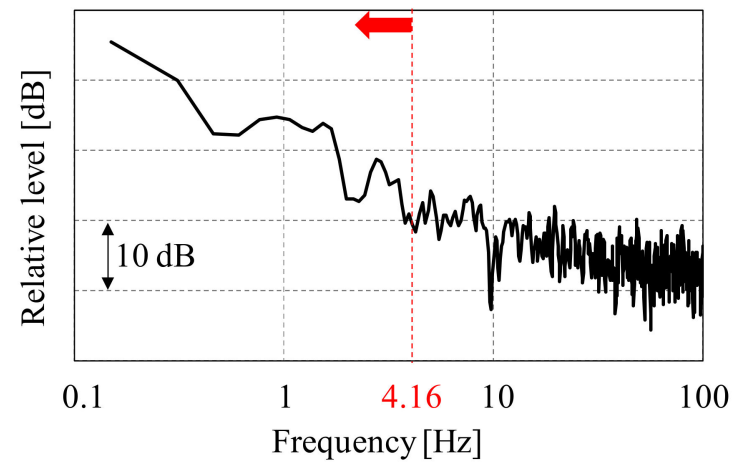

Figure 20. Frequency component of the fluctuation in the time-series data of vibration of the lifter under the normal condition. 


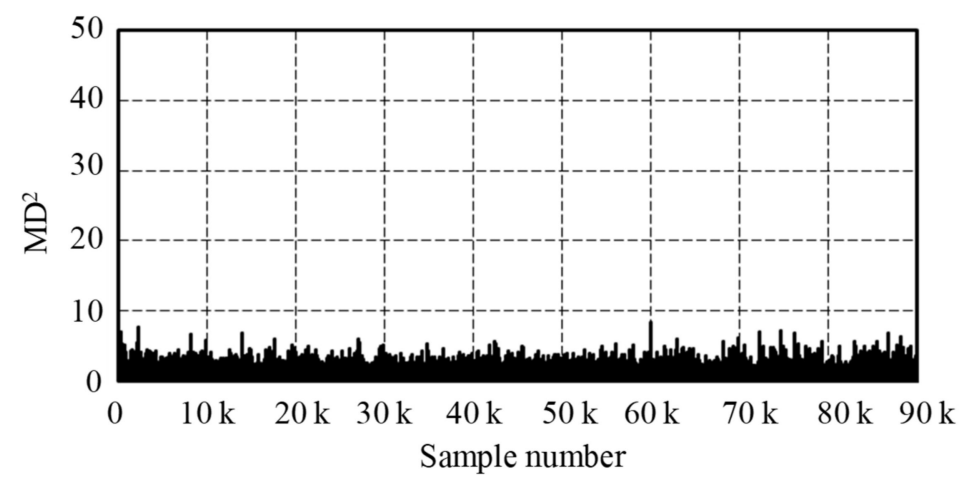

Figure 21. $\mathrm{MD}^{2}$ of the time-series data under the normal condition: In this result, the trends of the $\mathrm{MD}^{2}$ values during the nine days of normal operation are removed by the filtering and appear to be relatively constant.

\subsubsection{Calculation of $\mathrm{MD}^{2}$ Under Damaged Conditions}

The $\mathrm{MD}^{2}$ values under the various damaged conditions were calculated based on the generated unit space and the filtered time series of the vibration under the damaged condition. Note that the time-series energy levels under each of the damaged conditions were also filtered by the same high-pass filter designed and were adopted for the normal cases in the previous section. The $\mathrm{MD}^{2}$ results based on over 30,000 data values obtained from the continuous three-day operation of the lifter for the cases of belt and guide wheel damage are shown in Figure 22a,b, respectively. The $\mathrm{MD}^{2}$ results from 10,000 data values obtained during a one-day operation for the case in which the bearings were loaded by a strong external force are given in Figure 22c. All of these results show an $\mathrm{MD}^{2}$ that is substantially larger than the threshold value of 3.32. Thus, it appears that the value of $\mathrm{MD}^{2}$ is severely affected by the various types of artificially induced damage examined in this study, at least to the extent that they were applied here.

(a) Normal condition

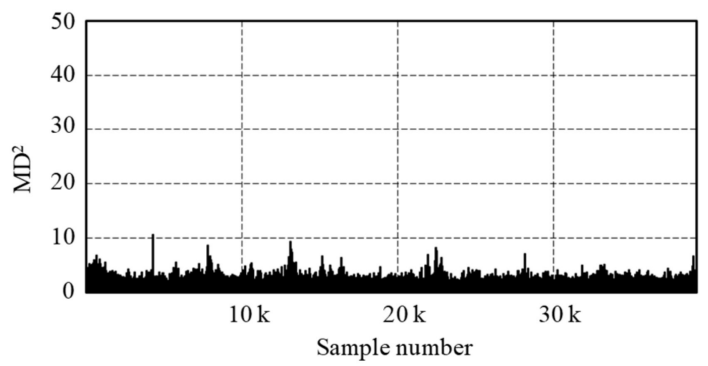

(c) Condition with damage on the belt

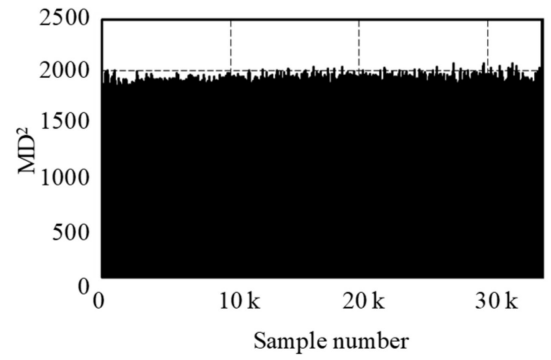

(b) Condition with damaged wheel

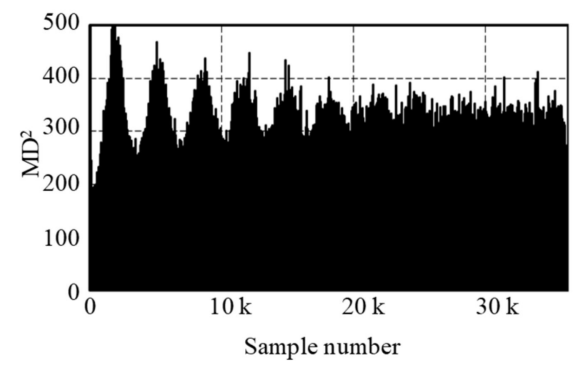

(d) Condition with damage on the bearing

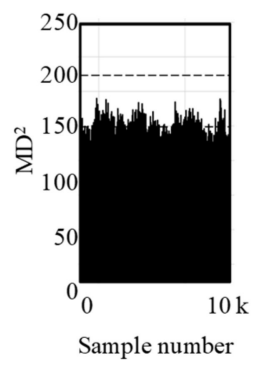

Figure 22. $\mathrm{MD}^{2}$ of the time-series data under the (a) normal condition and conditions damaged with the (b) guide wheel, (c) belt, and (d) bearing, respectively: These results in all of damaged three conditions from Figure $22 \mathbf{b}-\mathbf{d}$ show an $\mathrm{MD}^{2}$ that is substantially larger than the threshold value of 3.32, and they are severely affected by the various types of artificially induced damage. 


\subsection{Time-Series Simulation of $M D^{2}$ Based on Long-Term Operation}

We simulated the transition of the $\mathrm{MD}^{2}$ values based on the measured vibration data collected from the continuous operation of the lifter over a period of two years, assuming that the mechanical condition of the guide wheel will change from the normal state to the anomaly state when damaged to the same extent as described in the previous section over this period. First of all, we have two kinds of vibration data in the normal and damaged conditions that does not include the intermediate transition data in the period between the normal and anomaly terms. Therefore, the transition data were simply simulated assuming that the energy level in each frequency band linearly decays. The required time for the value of $\mathrm{MD}^{2}$ to exceed the anomaly threshold value was estimated based on the simulated transition. In this case study, we sought to validate that high-pass filtering can remove the short-term periodic trend but can preserve the long-term transition of the $\mathrm{MD}^{2}$ that appears as a gradual increase in the estimated values over several years. As reported earlier, the short-term periodic components that result in the unnecessary trend observed in the vibration time-series data were removed by high-pass filtering; however, we wanted to validate its usefulness in a practical case involving the long-term continuous observation of $\mathrm{MD}^{2}$.

Figures 19a and 23 show the time-series energy levels in each frequency band under normal and anomaly (guide wheel damage) conditions, respectively. Here, the transition of the energy levels from the normal condition (Figure 19a) to the anomaly condition involving damage to the guide wheel (Figure 23) was simulated to linearly change from one state to the other when operating the lifter continuously for 780 days. Note that, for the time-series data in the normal condition, 90 days were added before the 780 days of operation to verify that the $\mathrm{MD}^{2}$ value in the normal condition was constant at around 1.0.

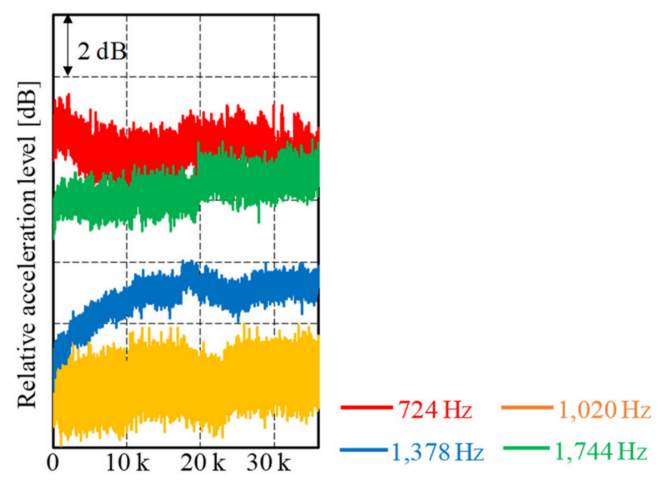

Figure 23. Vibration time-series data under the damaged-bearing condition: In this simulation study, the transition of the energy levels from the normal condition (Figure 19a) to this anomaly condition is simulated to linearly change from one state to the other when operating the lifter continuously for 780 days.

The transition of the time-series energy levels during the simulated 780 days was generated as follows. First, the data for the normal condition for the eight days that were described in Section 4.3.1 were combined repeatedly. The steady-state time-series data for 780 days was then linearly decreased to smoothly connect to the energy levels under the condition of a damaged guide wheel. As an example, the data for the $1378 \mathrm{~Hz}$ band are shown in Figure 24. In these data, the energy level decreases monotonically by approximately $5 \mathrm{~dB}$ over the 780-day period. Then, as shown in Figure 25, by using the time-series data of the first five days (50,460 data values), the unit space was generated. Next, the 50,460 values of $\mathrm{MD}^{2}$ for the five days beginning on the second day were calculated and the average value, $\overline{\mathrm{MD}}_{1}{ }^{2}$, was treated as the first $\mathrm{MD}^{2}$ data value. This operation was performed in the same manner for all of the data simulated over 870 days, each time advancing the five-day range by one day. The resulting $\overline{\mathrm{MD}}_{i}{ }^{2}(1 \leq i \leq 866)$ values are given in Figure 26a. As shown in the figure, 
the value of $\overline{\mathrm{MD}}^{2}$ changes as the energy level changes from the normal state to the anomaly state. The $\overline{\mathrm{MD}}^{2}$ for the first 200 days is shown in Figure 26b. The threshold value of 3.3 is also shown.

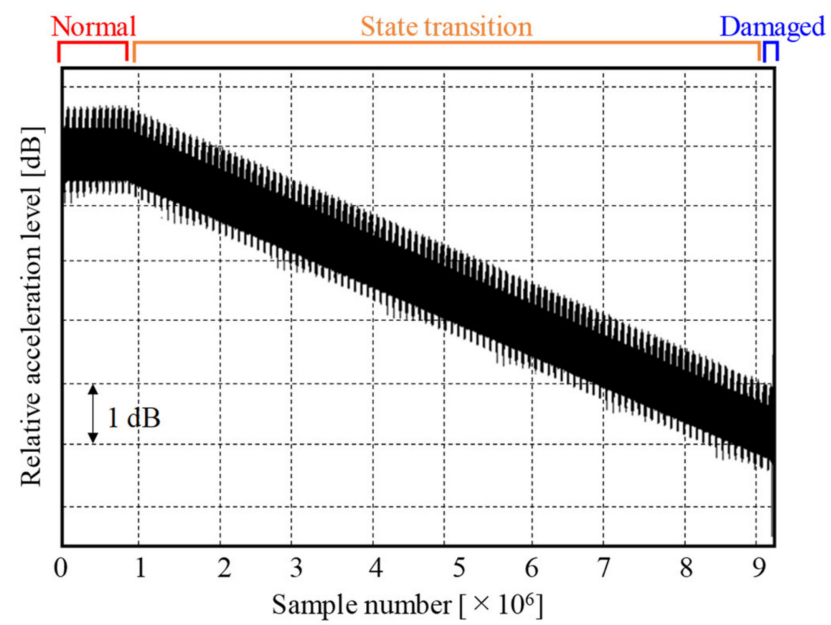

Figure 24. Simulated time-series data for vibration in the $1378 \mathrm{~Hz}$ band over 870 days: In these data, the energy level decreases monotonically by approximately $5 \mathrm{~dB}$ over the 780-day period.

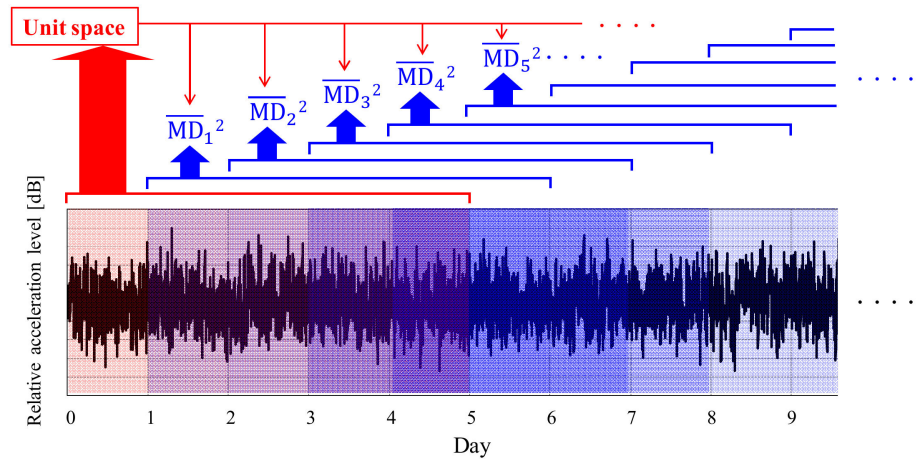

Figure 25. Calculation of $\mathrm{MD}^{2}$ for each day in the 870-day simulation: By using the time-series data of the first five days, the unit space is generated. Next, the 50,460 values of $\mathrm{MD}^{2}$ for the five days beginning on the second day were calculated as $\overline{\mathrm{MD}}_{1}{ }^{2}$. This operation is performed in the same manner for all of the data of 870 days.

(a) Entire transition of $\mathrm{MD}^{2}$

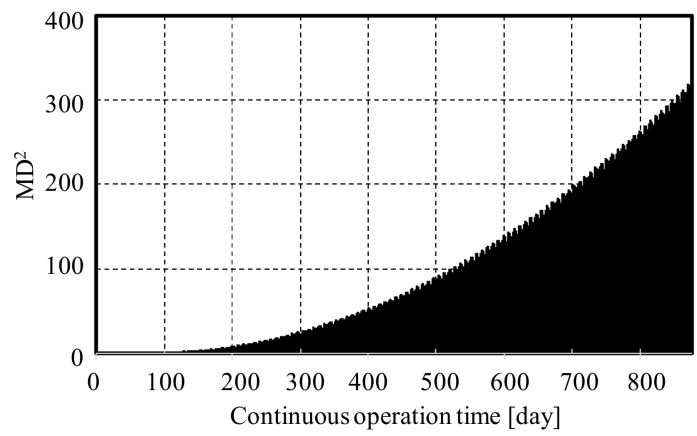

(b) Transition of $\mathrm{MD}^{2}$ in the early days

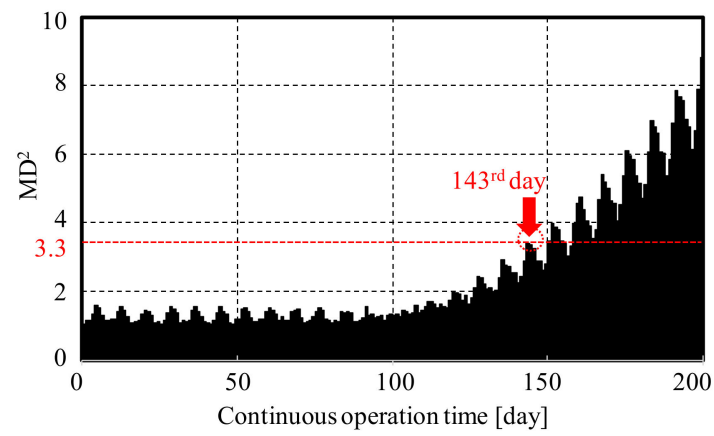

Figure 26. Transition of $\mathrm{MD}^{2}$ in the simulated time-series data (a) over the entire 870 days and (b) during the early 200 days of operation: As shown in Figure 26a, the value of $\overline{\mathrm{MD}}^{2}$ changes as the energy level changes from the normal state to the anomaly state. At the 143rd day from the beginning of the data in Figure 26b, $\overline{\mathrm{MD}}^{2}$ exceeds the threshold value of 3.3. 
The values of $\overline{\mathrm{MD}}^{2}$ do not appear to change from approximately 1.0 through the first 90 days of operation, as the state during this period had not changed. However, the value of $\overline{\mathrm{MD}}^{2}$ starts to rise from the 91st day due to the changing state of the device to the anomaly condition; 53 days later (i.e., 143 days from the beginning of the data), $\overline{\mathrm{MD}}^{2}$ exceeds the 3.3 threshold. Thus, based on the results of our simulation, where we assumed that, over the course of 780 days, the normal state would change to an anomaly state in which the value of $\mathrm{MD}^{2}$ is approximately 300 (Figure 22c), we were able to detect at quite an early point-in fact, within the first $7 \%$ of the total elapsed time- that an anomaly had occurred.

\section{Conclusions}

An approach for detecting anomalies in a logistic operating system using the MT method was proposed. In this approach, the frequency characteristics of vibration acceleration are used as multivariate parameters. The approach was validated through experimental studies involving an automated cargo lifter.

The applicability of the method for steady-state vibrations generated from a simple shaker was first examined. A steady-state signal superimposed with pure tones at 11 frequencies was output from the shaker, and $\mathrm{MD}^{2}$ values were calculated from the resulting vibration acceleration data. It was confirmed that the $\mathrm{MD}^{2}$ values for the measured acceleration calculated using the unit space generated from the same data had an average of 1.0 when the state of the installation of the shaker was unchanged. However, it should be noted that only the frequency components in frequency ranges having predominant relative levels such as the modal peak frequencies should be used in the analysis. Furthermore, moderate cycle fluctuations should be removed from the time-series data by using high-pass filtering.

Based on the above findings, the properties of the lifter in normal and artificially damaged conditions were investigated. $\mathrm{MD}^{2}$ values for fragment data acquired under three types of anomaly conditions were calculated based on the unit space generated from the fragment data obtained from the measurement results under the normal condition over nine days. In the normal condition, the $\mathrm{MD}^{2}$ values showed an average of 1.0. In contrast, in the anomaly conditions, the $\mathrm{MD}^{2}$ values substantially exceeded the threshold value of 3.3 (determined using a probability level of 0.01 in a $\chi^{2}$ distribution with 4 degrees of freedom). This result suggests that the operating state of the lifter can be estimated by using the differences in $\mathrm{MD}^{2}$ calculated from the frequency characteristics of the vibration.

Finally, the transition of the mechanical condition of the lifter was simulated over 870 days by using the collected measurement data in this paper and was used for the validation of the proposed method. Since we had two kinds of measured vibration data in the normal and damaged conditions that does not include the intermediate transition data in the period between the normal and anomaly terms, the transition data were simply simulated assuming that the energy level in each frequency band decays linearly. Based on this simulation in which the lifter was continuously operated for a period of approximately two years and the mechanical condition of the lifter's guide wheel changed gradually from its normal state to the anomaly condition, the transition of $\mathrm{MD}^{2}$ was predicted based on the simulated transition of the fragment data. It was observed that the $\mathrm{MD}^{2}$ value gradually increased in response to changes in the vibration characteristics over 780 days. As a result, we confirmed that the transition to the anomaly condition could be detected quite early in the process.

As a future work, the proposed method will be validated if the transition of the mechanical condition of the target lifter can be accurately detected by the method in a situation in which the lifter is continuously operated for over years. Furthermore, the applicability of the proposed method will be confirmed in more practical and complicated cases in which the lifter is operated with many loading products in a real distribution factory. After these validations are confirmed, the proposed method will make it possible to accurately monitor the transition of the mechanical conditions of the logistic systems. Therefore, the field of application of the detection method will be expanded to monitor the 
conditions of more general mechanical objects that provide regular mechanical movements, such as the robotics devices for production machine in the factories.

Author Contributions: Conceptualization, T.A.; data curation, W.Y.; formal analysis, T.A. and K.S.; funding acquisition, T.A. and M.S.; investigation, T.A.; project administration, T.A., K.S. and M.S.; resources, M.S.; software, W.Y. and K.S.; supervision, K.S. and M.S.; writing-original draft, T.A.; writing-review \& editing, K.S. and M.S. All authors have read and agreed to the published version of the manuscript.

Funding: This research received no external funding.

Conflicts of Interest: The authors declare no conflict of interest.

\section{References}

1. Krishnanunni, C.G.; Raj, R.S.; Nandan, D.; Midhun, C.K.; Sajith, A.S.; Ameen, M. Sensitivity-based damage detection algorithm for structures using vibration data. J. Civ. Struct. Health Monit. 2019, 9, $137-151$. [CrossRef]

2. Jin, X.; Sun, T.; Que, Z.; Wang, Y.; Chow, T.W.S. Anomaly Detection and Fault Prognosis for Bearings. IEEE Trans. Instrument. Measure. 2016, 65, 2046-2054. [CrossRef]

3. Cui, Y.; Bangalore, P.; Tjernberg, L.B. An anomaly detection approach using Wavelet transform and artificial neural networks for condition monitoring of wind turbines' Gearboxes. In Proceedings of the 2018 Power Systems Computation Conference (PSCC), Dublin, Ireland, 11-15 June 2018; pp. 1-7.

4. Korbicz, J.; Kowalczuk, Z.; KoScielny, J.M.; Cholewa, W. Fault Diagnosis; Springer: Berlin, Germany, 2004.

5. Hensman, J.; Worden, K.; Eaton, M.; Pullin, P.; Holford, K.; Evans, S. Spatial scanning for anomaly detection in acoustic emission testing of an aerospace structure. Mech. Syst. Signal Process. 2011, 25, 2462-2474. [CrossRef]

6. Li, W.; Zhu, Z.; Jiang, F.; Zhou, G.; Chen, G. Fault diagnosis of rotating machinery with a novel statistical feature extraction and evaluation method. Mech. Syst. Signal Process. 2015, 50-51, 414-426. [CrossRef]

7. Iannace, G.; Ciaburro, G.; Trematerra, A. Fault diagnosis for UAV blades using artificial neural network. Robotics 2019, 8, 59. [CrossRef]

8. Zhou, H.; Chen, J.; Dong, G.; Wang, R. Detection and diagnosis of bearing faults using shift-invariant dictionary learning and hidden Markov model. Mech. Syst. Signal Proccess. 2016, 72-73, 65-79. [CrossRef]

9. Li, B.; Zhang, P.; Tian, H.; Mi, S.; Liu, D.; Ren, G. A new feature extraction and selection scheme for hybrid fault diagnosis of gearbox. Exp. Syst. Appl. 2011, 38, 10000-10009. [CrossRef]

10. Assaad, B.; Eltabach, M.; Antoni, J. Vibration based condition monitoring of a multistage epicyclic gearbox in lifting cranes. Mech. Syst. Signal Process. 2014, 42, 351-367. [CrossRef]

11. Agrawal, S.; Agrawal, J. Survey on anomaly detection using data mining techniques. Proced. Comput. Sci. 2015, 60, 708-713. [CrossRef]

12. Iannace, G.; Ciaburro, G.; Trematerra, A. Heating, Ventilation, and Air Conditioning (HVAC) Noise Detection in Open-Plan Offices Using Recursive Partitioning. Buildings 2018, 8, 169. [CrossRef]

13. Iannace, G.; Ciaburro, G.; Trematerra, A. Wind Turbine Noise Prediction Using Random Forest Regression. Machines 2019, 7, 69. [CrossRef]

14. Nasseri, A.; Mohammadzadeh, M.J.; Tabatabaei Raeisi, S.H. Fracture enhancement based on artificial ants and fuzzy C-means clustering (FCMC) in Dezful Embayment of Iran. J. Geophys. Eng. 2015, 12, 227-241. [CrossRef]

15. Zhang, D. Crack detection for bituminous pavements based on cluster and minimum spanning tree. Acta Sci. Nat. Univ. Sunyats. 2017, 4, 68-74.

16. Taguchi, G.; Chowdhury, S.; Wu, Y. The Mahalanobis-Taguchi System; McGraw-Hill: New York, NY, USA, 2001.

17. Taguchi, G.; Rajesh, J. New Trends in Multivariate Diagnosis. Sankhyā Indian J. Stat. Ser. B 2000, 62, $233-248$.

18. Soylemezoglu, A.; Sarangapani, J.; Saygin, C. Mahalanobis Taguchi system (MTS) as a prognostics tool for rolling element bearing failures. J. Manuf. Sci. Eng. 2010, 132, 051014. [CrossRef]

19. Shakya, P.; Kulkarni, M.S.; Darpe, A.K. A novel methodology for online detection of bearing health status for naturally progressing defect. J. Sound Vib. 2014, 333, 5614-5629. [CrossRef]

20. Balsamo, L.; Betti, R.; Beigi, H. A structural health monitoring strategy using cepstral features. J. Sound Vib. 2014, 33, 4526-4542. [CrossRef] 
21. Piñeyro, J.; Klempnow, A.; Lescano, V. Effectiveness of new spectral tools in the anomaly detection of rolling element bearings. J. Alloys Compd. 2000, 310, 276-279. [CrossRef]

22. Barszcz, T.; Randall, R.B. Application of spectral kurtosis for detection of a tooth crack in the planetary gear of a wind turbine. Mech. Syst. Signal Process. 2009, 23, 1352-1365. [CrossRef]

23. Yang, Y.; Miao, C.; Li, X.; Mei, X. On-line conveyor belts inspection based on machine vision. Optik 2014, 125, 5803-5807. [CrossRef]

24. Li, J.; Miao, C. The conveyor belt longitudinal tear on-line detection based on improved SSR algorithm. Optik 2016, 127, 8002-8010. [CrossRef]

25. Hou, C.; Qiao, T.; Zhang, H.; Pang, Y.; Xiong, X. Multispectral visual detection method for conveyor belt longitudinal tear. Measurement 2019, 143, 246-257. [CrossRef]

26. Kirjanow-Blazej, A.; Jurdziak, L.; Burduk, R.; Blazej, R. Forecast of the remaining lifetime of steel cord conveyor belts based on regression methods in damage analysis identified by subsequent DiagBelt scans. Eng. Fail. Anal. 2019, 100, 119-126. [CrossRef]

27. Mendenhall, W.; Sincich, T. Statistics for Engineering and the Sciences, 5th ed.; Pearson/Prentice Hall: Upper Saddle River, NJ, USA, 2007.

(C) 2020 by the authors. Licensee MDPI, Basel, Switzerland. This article is an open access article distributed under the terms and conditions of the Creative Commons Attribution (CC BY) license (http://creativecommons.org/licenses/by/4.0/). 